\title{
REVIEW ARTICLE Mechanical properties of Fe-rich Si alloy from Hamiltonian
}

\author{
Tetsuo Mohri ${ }^{1}$, Ying Chen ${ }^{2}$, Masanori Kohyama ${ }^{3}$, Shigenobu Ogata $\mathbb{C}^{4,5}$, Arkapol Saengdeejing ${ }^{2}$, Somesh Kumar Bhattacharya ${ }^{3,6}$,
} Masato Wakeda $\mathbb{D D}^{4}$, Shuhei Shinzato ${ }^{4}$ and Hajime Kimizuka ${ }^{4}$

The physical origins of the mechanical properties of Fe-rich Si alloys are investigated by combining electronic structure calculations with statistical mechanics means such as the cluster variation method, molecular dynamics simulation, etc, applied to homogeneous and heterogeneous systems. Firstly, we examined the elastic properties based on electronic structure calculations in a homogeneous system and attributed the physical origin of the loss of ductility with increasing Si content to the combined effects of magneto-volume and $\mathrm{DO}_{3}$ ordering. As a typical example of a heterogeneity forming a microstructure, we focus on grain boundaries, and segregation behavior of $\mathrm{Si}$ atoms is studied through high-precision electronic structure calculations. Two kinds of segregation sites are identified: looser and tighter sites. Depending on the site, different segregation mechanisms are revealed. Finally, the dislocation behavior in the Fe-Si alloy is investigated mainly by molecular dynamics simulations combined with electronic structure calculations. The solid-solution hardening and softening are interpreted in terms of two kinds of energy barriers for kink nucleation and migration on a screw dislocation line. Furthermore, the clue to the peculiar work hardening behavior is discussed based on kinetic Monte Carlo simulations by focusing on the preferential selection of slip planes triggered by kink nucleation.

npj Computational Materials (2017)3:10 ; doi:10.1038/s41524-017-0012-4

\section{INTRODUCTION}

Mechanical properties of alloys originate from the behavior of electrons and atoms on the microscopic scale; however, the strength of atomic bonding does not directly relate to macroscopic mechanical properties such as the yield strength, fracture toughness, and fatigue resistance. This is because the microstructure on the mesoscale, which is composed of various defects such as impurities, grain boundaries, and dislocations, mediates or enhances the response of alloys against external forces, leading to fairly nonlinear and multiscale phenomena. Facing such a nonlinear nature associated with the strength and plastic deformation of alloys, identifying the controlling factors for the mechanical properties is a significantly difficult task, and clarifying the underlying physics at different length and time scales still remains a major challenge in materials science.

The authors of the present article took up the challenge of this complicated problem with their particular theoretical and computational tools unique to each characteristic scale range. These tools are electronic structure calculations, the cluster variation method (CVM) of statistical mechanics, and molecular dynamics (MD) simulations, which may cover the atomic to mesoscopic scale ranges. By virtue of high-performance computers, some of the results obtained by the present calculations achieve a higher precision and reveal a clearer picture than those obtained by ordinary experiments.

Among the various mechanical properties, the main focus of the present study is the solid-solution strengthening of Fe-rich Si alloys. In fact, the strength and plastic deformation properties of dilute alloys change dramatically by adding small amounts of alloying elements in many cases, even at concentrations below a few atomic percent. In general, the dissolved impurity atoms are homogeneously dispersed in the dilute alloy and the average elastic properties of the whole system often change monotonically, yet non-monotonic behavior takes place gradually or abruptly around or at a certain concentration of a solute element. Revealing the physical mechanism of such behavior is mainly the subject of electronic structure calculations, which clarify the origin in the density of states (DOS), and statistical mechanics calculations, which examine the configurational behavior of atoms. The second section is designated to this discussion.

For practical alloys that are, in general, polycrystalline states, the existence of grain boundaries plays a significant role in various mechanical properties in many ways. Among them is the disturbance of the homogeneous distribution of solute atoms in the matrix. In fact, solute elements segregate at grain boundaries and may cause a local change in the stress distribution at the grain boundary, which is not easily detected by ordinary experimental or theoretical techniques. In the third section, the segregation behavior at the atomic scale is revealed by high-precision electronic structure calculations applied mainly for coincidence grain boundaries.

Plastic deformation properties are exclusively attributed to dislocation behavior. In fact, the strength of the system often changes unpredictably and can exhibit values higher than those estimated with a linear scaling to the impurity concentration. This is the case when the solute element is localized in the vicinity of

\footnotetext{
${ }^{1}$ Institute for Materials Research, Tohoku University, Sendai 980-8577, Japan; ${ }^{2}$ Fracture and Reliability Research Institute, School of Engineering, Tohoku University, Sendai 9808579, Japan; ${ }^{3}$ Research Institute of Electrochemical Energy, Department of Energy and Environment, National Institute of Advanced Industrial Science and Technology, Ikeda,

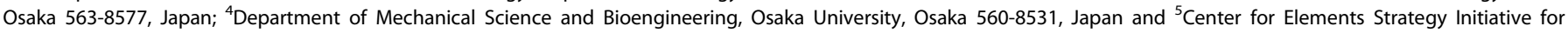
Structural Materials, Kyoto University, Kyoto 606-8501, Japan

Correspondence: Tetsuo Mohri (tmohri@imr.tohoku.ac.jp)

${ }^{6}$ Present address: Computational Structural Materials Design Group, Research Center for Structural Materials, National Institute for Materials Science, Tsukuba 305-0047, Japan
}

Received: 23 September 2016 Revised: 20 January 2017 Accepted: 25 January 2017

Published online: 10 March 2017 
dislocations and interacts through their elastic or electric fields. It is quite a difficult task to observe single dislocation behavior even with a high-resolution electron microscope. The recent development of MD simulations reveals the atomic processes of dislocation behavior at a given atomic configuration with the existence of solute elements. By combining such microscopic energetic processes experienced by a dislocation line with macroscopic phenomenological theory, the dependences of the mechanical properties on temperature, strain rate, etc. can be obtained for a different set of concentrations and configurations of solute atoms. This way, single dislocation behavior in a solid solution can be correlated with the experimental results of solidsolution hardening. The detailed calculations are reported in the fourth section.

From the various alloy systems, Fe-rich Si alloy was selected for the present study. Fe-rich Si alloy forms a solid solution up to $\sim 10$ at.\% $\mathrm{Si}$ at room temperature and exhibits various intriguing mechanical properties. ${ }^{1,2}$ For example, it was reported that the yield stress increased by $40 \%$ by the addition of only $1.0 \mathrm{wt} . \%$ (1.9 at.\%) $\mathrm{Si}^{3}{ }^{3}$ Also, the accompanying increase in the maximum tensile stress due to the $\mathrm{Si}$ addition resulted in a significant increase in the work hardening coefficient. ${ }^{4}$ The physical origins of all these phenomena, however, have not been fully resolved yet.

Another reason to select Fe-rich Si alloy is that this is a solid solution with a highly symmetric body-centered cubic (bcc) structure, which is the simplest possible phase to which various theoretical tools can be applied. In view of the fact that the multiscale calculations for mechanical properties are still in their infancy, it was deemed necessary for the theoretical tools to examine the reproducibility of commonly accepted mechanisms on a phase as simple as possible. The present study of the Fe-rich Si system is therefore to be regarded as a precursor for future sophisticated multiscale calculations.

\section{ELASTIC PROPERTIES}

Calculation procedure

Prior to the introduction of defects, it is essential to investigate the elastic and thermodynamic properties of a homogeneous system. Hence, we have conducted comprehensive first-principles total energy calculations on $\mathrm{Fe}-\mathrm{Si}$ alloy for the Si concentration range of interests. Electronic structure calculations to investigate the elastic properties of the system in the ground state were based on density functional theory (DFT) within the framework of generalized gradient approximation (GGA)-projector augmented wave method (PBE)-PAW implemented in VASP. ${ }^{5-8}$ All structures are fully relaxed, and a 300-400 eV cutoff energy and 5000-6000 kpoints meshes were adopted to provide sufficient convergence of the electronic energies for all calculations. The cluster expansion method $(\mathrm{CEM})^{9},{ }^{10}$ was employed together with electronic structure calculations to explore the stable phases in the ground state over the entire range of concentration. To clarify the origin of the change in the elastic properties in the concentration range near the phase boundary, the $\mathrm{CVM}^{11,12}$ was used in combination with DFT to extend the calculations into finite temperature range of $100-700 \mathrm{~K}$.

Elastic constants were calculated from completely relaxed structures using the stress-strain method based on the procedure proposed by Le Page and Saxe. ${ }^{13}$ The bulk $(B)$, shear $(G)$, and Young's $(E)$ moduli were evaluated using Voigt's method. ${ }^{14}$ To examine the concentration dependences of the properties at different Si concentrations, various supercells were constructed by adjusting the configurations and numbers of substitutional $\mathrm{Si}$ atoms within the Fe cell. The concentration of Si studied in the present report ranged from 2.8 to 12.5 at.\% as described in ref. 1 . In the majority of cases, a $4 \times 4 \times 4$ bcc primitive supercell was used.

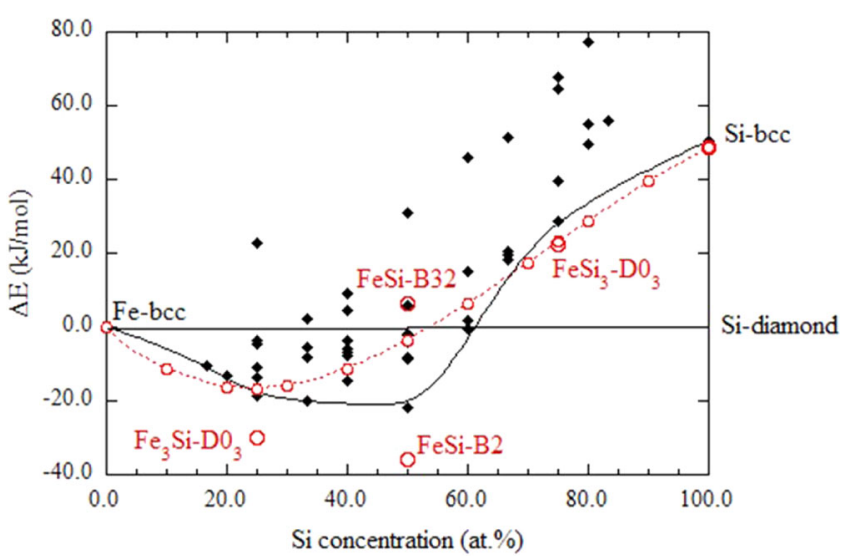

Fig. 1 Formation energies of a selected set of bcc-based structures and a random solid solution over the whole Si concentration range based on systematic ground state analysis using CEM for a large number of ordered structures in Fe-Si (black), and the corresponding results using a minimum set of 6 bcc-based structures as an approximation (red)

Energetics of homogeneous Fe-Si system; phase stability and elastic properties

Figure 1 shows the formation energies of a selected set of bccbased structures and a random solid solution for the whole $\mathrm{Si}$ concentration range evaluated by CEM on all these structures (black dots and line). The convex linking the minimum energies at the concentrations of $\mathrm{Fe}_{5} \mathrm{Si}(\mathrm{C2} / \mathrm{m}), \mathrm{Fe}_{3} \mathrm{Si}\left(\mathrm{DO}_{3}\right), \mathrm{Fe}_{2} \mathrm{Si}(\mathrm{Cmmm}), \mathrm{FeSi}$ (B2), $\mathrm{FeSi}_{2}\left(\mathrm{C}_{1} 1_{\mathrm{b}}\right)$, and $\mathrm{FeSi}_{3}(\mathrm{Pmmm})$ gives stable bcc-based structures as the ground state. In the same figure, the formation energy of a random solid solution evaluated from the cluster expansion on a set of 6 bcc-based structures (Fe-bcc, $\mathrm{Fe}_{3} \mathrm{Si}-\mathrm{DO}_{3}$, $\mathrm{FeSi}-\mathrm{B} 2, \mathrm{~B} 32, \mathrm{FeSi}_{3}-\mathrm{DO}_{3}$, and $\mathrm{Si}-\mathrm{bcc}$ ) is also plotted as the red line. One can see that on the Fe-rich side, the formation energies evaluated from this minimum set of bcc-based structures are close to the values (black line) calculated from the expansion using a larger number of structures, indicating that this set of 6 bcc-based structures could be used as a first-order approximation in the discussion of the dilute Si concentration, which is the main target of the present work. In fact, these 6 bcc-based structures have been used to extract the effective cluster interaction energies from DFT internal energies for the finite temperature calculations by CVM.

One of the most intriguing facts in $\mathrm{Fe}-\mathrm{Si}$ is an obvious degradation in the ductility reported for Si concentrations beyond 9-11 at.\%. ${ }^{15-17}$ This phenomenon, which is akin to a ductile-tobrittle transition (with concentration), has been well-known for a long time, yet to the best of the authors' knowledge a thorough understanding at the electronic structure scale has not been attempted.

Figure 2(a) shows the calculated ground-state elastic properties including the elastic constants $\left(C_{11}, C_{12}, C_{44}\right)$, bulk modulus $(B)$, shear modulus $(G)$, and Young's modulus $(E)$ at Si concentrations of 2.8, 5.6, 8.3, 9.4, 10.9, and 12.5 at.\%. ${ }^{1,18,19}$ The numerical values are listed in Table 1. One can see a steady change in all these constants with increasing Si concentration up to 9.4 at.\%, and an obvious decrease in all the constants takes place when the $\mathrm{Si}$ concentration exceeds 9.4 at.\%. These values reach minima at 10.9 at.\%Si, and then rebound to a certain extent as the $\mathrm{Si}$ concentration increases further. This trend is consistent with the experimental results. For example, the report of a measurement of $C_{11}$ at $0-25$ at.\%Si in ref. 16 shows that the value of $C_{11}$ decreases gradually from pure $\mathrm{Fe}$ to 11 at.\%Si and then rebounds monotonously to 25 at.\%Si, which coincides quite well with our 
calculated $C_{11}$ indicated by the red line in Fig. 2(a). Taking a closer look at the details of the $\mathrm{Si}$ concentration dependence of each property, one might find that properties relating to the tension $\left(C_{11}, C_{12}, E\right.$, and $\left.B\right)$ change obviously, while those properties relating to shearing ( $C_{44}$ and $G$ ) show a very gentle change, which implies that the ductility of the Fe-rich $\mathrm{Si}$ is dominated mainly by $B$. We then performed an analysis based on Pugh's empirical rule, $^{20}$ indicating that a $B / G$ ratio of 1.75 can be taken as a critical

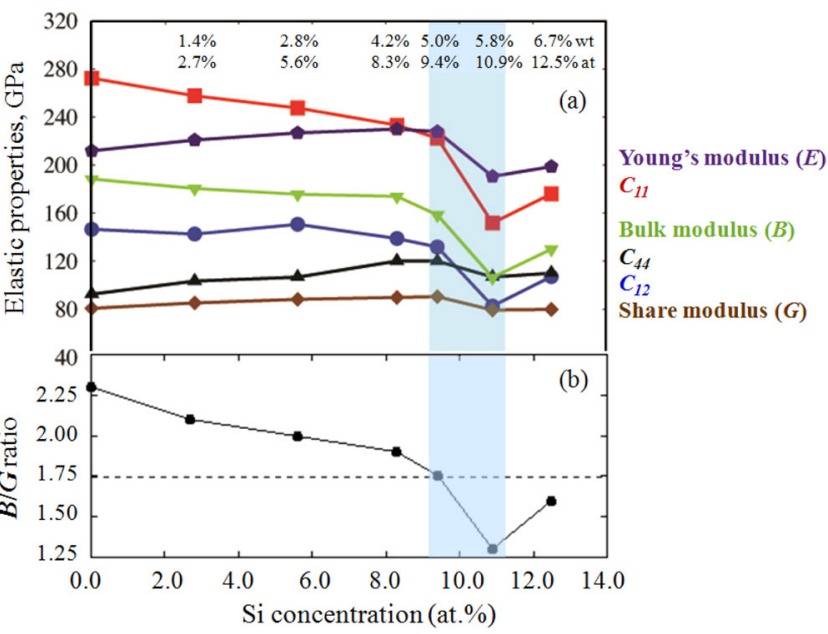

Fig. 2 a Calculated elastic properties in $0.0-12.5$ at.\%Si including elastic constants $\left(C_{11}, C_{12}, C_{44}\right)$, bulk modulus $(B)$, shear modulus $(G)$, and Young's modulus (E). ${ }^{1,} 18,19$ The $\mathrm{Si}$ concentrations in the superells used in the calculations are displayed at the top of the figure in both atomic percent age and weight percentage. The numerical values are listed in Table 1 . $\mathbf{b}$ The ratios of bulk modulus to shear modulus $(B / G)$ in $0.0-12.5$ at.\%Si using corresponding values in the Fig. 2(a)

Table 1. Elastic properties [GPa] in dilute Si iron-silicon alloys and comparison between systems with and without Ni-doping at 10.9 at.\% Si

\begin{tabular}{llrrrlll}
\hline Si concentration at.\%Si & \multicolumn{1}{l}{$C_{11}$} & \multicolumn{1}{c}{$C_{12}$} & \multicolumn{1}{c}{$C_{44}$} & \multicolumn{1}{l}{$B$} & \multicolumn{1}{l}{$G$} & \multicolumn{1}{l}{$B$} \\
\hline 0.0 & 272.3 & 146.5 & 92.5 & 188.4 & 80.7 & 211.8 & 0.5 \\
2.8 & 257.6 & 142.5 & 103.4 & 180.5 & 85.2 & 220.8 & 0.6 \\
5.6 & 247.5 & 150.6 & 106.7 & 175.7 & 88.2 & 226.7 & 0.6 \\
8.3 & 233.2 & 138.8 & 120.1 & 173.9 & 89.8 & 229.9 & 0.7 \\
9.4 & 222.3 & 131.8 & 120.0 & 158.5 & 90.4 & 227.8 & 0.8 \\
10.9 & 151.7 & 82.6 & 106.8 & 106.4 & 79.3 & 190.5 & 1.0 \\
12.5 & 176.0 & 107.1 & 110.0 & 130.0 & 79.8 & 198.7 & 0.8 \\
Ni-doped 10.9 & 193.3 & 122.2 & 109.3 & 145.8 & 79.8 & 202.4 & 1.8 \\
\hline
\end{tabular}

value between ductile $(>1.75)$ and brittle $(<1.75)$ states for metals. It can be noticed in Fig. 2(b) that the $B / G$ of our result demonstrates that 9.4 at.\% $\mathrm{Si}$ is right at the critical value between the ductile and brittle territories.

We note that the $B / G$ ratio is an indicator that provides a rough estimation of the intrinsic ductility/brittleness of a material. To predict the ductile-brittle transition temperature (DBTT), we need to calculate the temperature-dependent critical resolved shear stress (CRSS) and compare it with the critical stress necessary for crack propagation, such as $K_{I C}$. The temperature-dependent CRSS can be calculated in the present framework by analyzing the temperature- and stress-dependent dislocation velocity using MD simulation and kinetic Monte Carlo (kMC), as we describe in section 4. However, as will be discussed in the next section, when the magnetism plays a significant role, MD-based modeling is still not powerful enough to provide a critical answer because of the difficulty in implementing the magnetic ordering effect in the interatomic potentials. Hence, the DBTT prediction of Fe-Si alloys with magnetic ordering effects is still a challenging task, and our analysis is taken as a precursor to a more sophisticated analysis of the temperature dependency.

Competition of magnetic and ordering effects

Our detailed analysis ${ }^{18,19}$ of the electronic structures revealed that the drop in the elastic properties over 9-11 at.\%Si originated from the instability at the Fermi energy, which is furthermore related to some particular configurations of $\mathrm{Fe}$ and $\mathrm{Si}$ atoms formed in the alloy. This point is also discussed ${ }^{18,19}$ in terms of the competition between the magneto-volume effect ${ }^{21}$ at low Si concentrations up to 9 at.\% and the effect of $\mathrm{DO}_{3}$ ordering at Si concentrations higher than 12 at.\% in the following way.

Firstly, we examined the evolution of the electronic structures with $\mathrm{Si}$ concentration in terms of the DOS. Figures 3(a) and (b) show the DOS of the systems at 9.4 and 10.9 at.\%Si, respectively. Paying attention to the value of the DOS at the Fermi level $\left(E_{f}\right)$, we see a big difference in the two cases at minority spin. In the case of 9.4 at.\% $\mathrm{Si}$, the Fermi level is at the dip of the two peaks, whereas in the case of 10.9 at.\%Si, it coincides with a big narrow peak. It is known that if the Fermi level of a certain configuration lies right at a large DOS peak, the system will become unstable because a slight shift in the DOS distribution results in a large variation in the electronic states at the Fermi surface.

To determine the origin of the peak, we investigated the local density of states (LDOS) of each Fe atom in the supercell at the Fermi level, $N_{n}\left(E_{f}\right)$, where $n(=1,2,3, \ldots$,$) indicates the position of$ atoms in the supercell as shown in Fig. 4(b). As plotted in Fig. 4(a), all the Fe atoms can be classified into three groups according to the contribution of $N_{n}\left(E_{f}\right)$ of each atom and the configuration of this atom with its first nearest-neighbor $(1 \mathrm{nn})$ atoms; the first group classifies Fe atoms with a high value of $N_{n}\left(E_{f}\right)>1.5$, which have two $1 \mathrm{nn} \mathrm{Si}$ atoms to form a linear $\mathrm{Si}-\mathrm{Fe}-\mathrm{Si}$ configuration; the second group are those with a low value of $N_{n}\left(E_{f}\right)<1.1$, which have no $\mathrm{Si}$ atoms as $1 \mathrm{nn}$; and the last group are those with an $N_{n}\left(\mathrm{E}_{\mathrm{f}}\right)$ value between the values of the first two groups and have

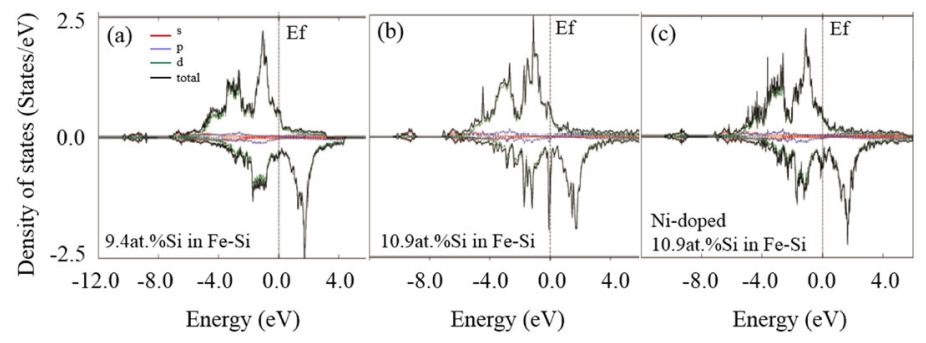

Fig. 3 Densities of states in three systems: a 9.4at.\%Si in Fe-Si, b 10.9at.\%Si in Fe-Si and c Ni-doped 10.9at.\%Si in Fe-Si 
(a)

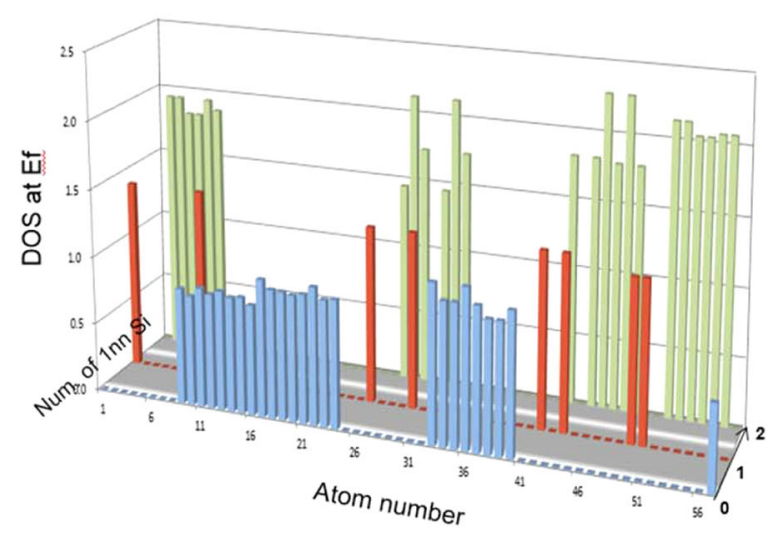

(b)

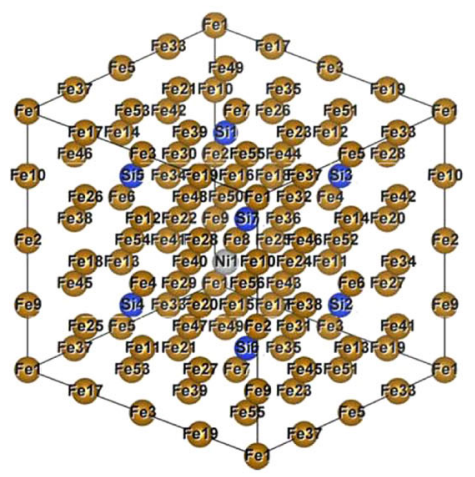

Fig. 4 Analysis of density of state at 10.9at.\%Si. a LDOS of all Fe atoms (1-57 in b) at Ef. Green indicates Fe atom with two $\mathrm{Si}$ in the $1 \mathrm{nn}$, red with one $\mathrm{Si}$ in the $1 \mathrm{nn}$ and blue without $\mathrm{Si}$ in the $1 \mathrm{nn}$. b Supercell model at 10.9 at. $\% \mathrm{Si}$

one $1 \mathrm{nn} \mathrm{Si}$ atom. This observation leads to an important fact that the largest contributions to $N\left(\mathrm{E}_{\mathrm{f}}\right)$ come from those linear $\mathrm{Si}-\mathrm{Fe}-\mathrm{Si}$ configurations, which revealed a direct correlation between the Fermi level stability and the atomic configurations. Since the force constants obtained from phonon vibrations ${ }^{1}$ show that $\mathrm{Si}$ in bccFe induces a repulsive interaction in the $1 \mathrm{nn}$ Fe-Si pairs, one could understand that such a repulsive interaction pushes the electrons to a higher energy to cause higher DOS values at the Fermi level, which should be the origin of the reduction in the elastic properties at 10.9 at.\%Si in the Fe-Si alloy.

To gain a thorough understanding of the mechanism of the $\mathrm{Si}$ concentration dependence of the elastic properties shown in Fig. 2, we summarized the total DOS at $E_{f}$ and the local magnetic moment for all the Si concentrations and further examined the relationship between the equilibrium volumes and average magnet moments. It was found ${ }^{1}$ that with increasing $\mathrm{Si}$ concentration, the magnetic moments decrease, which leads to a reduced equilibrium volume as a consequence of negative magnetic pressure. Such a magnetic-moment-induced volume change is known as the magneto-volume effect. ${ }^{21}$ Taking $B$ as an example of the seven elastic properties under consideration, we evaluated the change in $B$ induced by magnetic moments using Stoner's model of itinerant magnetism ${ }^{22}$ and revealed that this model explains well the Si concentration dependence of $B$ up to 9.4 at.\% $\mathrm{Si}$, but it cannot interpret the drastic change in $B$ as the $\mathrm{Si}$ concentration increases above 9.4 at.\% $\mathrm{Si}^{1}{ }^{1}$

In the range of higher Si concentrations from 10.9 to 12.5 at.\%Si, there should be another reason behind the recovery of the elastic properties as displayed in Fig. 2. Referring to the latest estimation of the Fe-Si phase diagram, ${ }^{23}$ and paying attention to the low- temperature region on the Fe-rich side, we notice that three bccbased structures, a-Fe, FeSi-B2, and $\mathrm{Fe}_{3} \mathrm{Si}_{2}-\mathrm{DO}_{3}$, co-exist in this region. The boundary of $\mathrm{a}-\mathrm{Fe}$ and $\mathrm{DO}_{3}$ is estimated to pass through $10-11$ at.\%Si for temperatures below $500^{\circ} \mathrm{C}$. This means that 12.5 at.\% $\mathrm{Si}$ is already in the domain of $\mathrm{DO}_{3}$, suggesting that the $\mathrm{DO}_{3}$ ordering effect should be considered.

For this, the CVM within the tetrahedron approximation ${ }^{12}$ combined with the effective cluster interaction energies derived based on the six bcc-based ordered structures set (Fe-bcc, $\mathrm{Fe}_{3} \mathrm{Si}-\mathrm{DO}_{3}, \mathrm{FeSi}-\mathrm{B2}, \mathrm{B} 32, \mathrm{FeSi}_{3}-\mathrm{DO}_{3}$, and $\mathrm{Si}-\mathrm{bcc}$ ) was employed, and the probabilities of six kinds of tetrahedron clusters in bccdisordered $\mathrm{Fe}-\mathrm{Si}$ were obtained for a temperature range of $100-700 \mathrm{~K}$. It is noted that when the Si concentration is lower than $25 \mathrm{at} \% \mathrm{Si}$, only two clusters are dominantly detected. One is composed of only Fe atoms corresponding to the Fe-bcc phase and the other is of three $\mathrm{Fe}$ atoms and a single $\mathrm{Si}$ atom corresponding to the $\mathrm{Fe}_{3} \mathrm{Si}-\mathrm{DO}_{3}$ phase; the probabilities of the other four kinds of clusters are almost zero. When the $\mathrm{Si}$ concentration increases from pure $\mathrm{Fe}$, the Fe-bcc-related cluster decreases, whereas the $\mathrm{Fe}_{3} \mathrm{Si}-\mathrm{DO}_{3}$-related cluster increases and becomes dominant above 12.5 at $\%$. We then estimated the concentration dependence of $B$ in terms of the contributions of the two dominant phases, which is a cluster expansion operated on $B$ at low temperatures such as $300 \mathrm{~K}$. Because of the higher probability of the $\mathrm{DO}_{3}$-related clusters beyond 12.5 at\%, together with the fact that $B^{\mathrm{D} 03}(223 \mathrm{GPa}, 0 \mathrm{~K})>B^{\mathrm{Fe}-\mathrm{bcc}}(188 \mathrm{GPa}, 0 \mathrm{~K}){ }^{24}$ one could ascribe the origin of the increase at the higher $\mathrm{Si}$ concentration to the ordering effect. Hence, it is concluded that the opposite concentration dependence induced by magnetovolume and ordering effects result in the non-monotonic behavior of $B$ discussed above.

Effect of Ni-doping based on electronic structures and elastic properties

Microscopic insights into the concentration dependence of the elastic properties in dilute Fe-Si alloy were obtained as discussed above, and in particular, the origin of the Fermi level instability was ascribed to the repulsive interactions of certain $\mathrm{Si}-\mathrm{Fe}-\mathrm{Si}$ configurations in the Fe-Si alloy at a specific Si concentration. Based on these findings, we conducted further calculations to investigate the effect of $\mathrm{Ni}$ doping in $\mathrm{Fe}-\mathrm{Si}$ as an attempt to improve the elastic properties, since the attractive feature of $\mathrm{Ni}$ in Fe-bcc ${ }^{25}, 26$ may moderate the electronic distribution at the Fermi level. Figure 3(c) shows the DOS of the system with one substitutional $\mathrm{Ni}$ atom in 10.9 at.\%Si, and it is amazing to find that the large peak at the minority-spin DOS of 10.9 at.\%Si nearly disappears upon Ni doping.

A detailed analysis of the $N_{n}\left(\mathrm{E}_{\mathrm{f}}\right)$ of all the Fe atoms in the case of $\mathrm{Ni}$ doping has been performed to obtain a figure similar to that of Fig. 4. It was found that all the local contributions to the $N_{n}\left(E_{f}\right)$ from all $\mathrm{Fe}$ atoms in the case of $\mathrm{Ni}$ doping decreased more or less compared with the case without $N i$ doping and that the $N_{n}\left(\mathrm{E}_{\mathrm{f}}\right)$ from those big "contributor" Fe atoms (marked in green in Fig. 4) dropped by $2 / 3$ of the original values in the undoped system. The last row in Table 1 shows the improvement in all seven elastic properties, especially for $C_{11}$ and $B$, compared with those in the undoped systems. In fact, it is already known from early experiments that high-Si-content steel needs a certain amount of $\mathrm{Ni}$ to improve the ductility, ${ }^{17}$ and the present results substantiate this. The mechanism of such $\mathrm{Ni}$-doping enhancement of the Fermi level stability can be understood as the result of attractive interactions introduced by $\mathrm{Ni}$ doping that shake out the high values of the DOS at the Fermi level so as to enhance the stability. This is an encouraging indication of tailoring electronic structures by introducing specific atomic configurations that adjust the bonding states and the distribution of electrons to enhance specific physical properties. 


\section{SEGREGATION AT GRAIN BOUNDARIES AND LOCAL ENERGY/ STRESS ANALYSES}

Producing homogeneous materials has been the main strategy of manufacturing industries. Contrary to this, one recent trend is to introduce heterogeneity at the microstructure scale under wellcontrolled processes. In both cases, understanding and controlling the heterogeneity are unique strategic factors particularly for alloy design. Among the typical heterogeneities found in alloys, the grain boundary (GB) is addressed in the present study. In particular, GBs disturb the homogeneous distribution of solute atoms in the matrix by attraction or ejection. This may cause local changes in the stress and elastic and magnetic properties at the GB. Although numerous attempts have been made to analyze the atomic segregation behavior, a detailed analysis including the resultant stresses and elastic and magnetic properties has not, to the best of the authors' knowledge, been attempted.

\section{Development of local-energy and local-stress schemes}

As a specific theoretical scheme to investigate local changes in various properties at GBs, the practical computational technique of local-energy and local-stress calculations based on DFT has been developed by the group of one of the authors [MK]. Since the theoretical framework has been amply demonstrated in previous reports, ${ }^{27,}{ }^{28}$ we merely summarize the essential points in the following.

In conventional plane-wave DFT schemes, the total energy and stress tensor are given as integrated or averaged quantities within the supercell, and we cannot gain knowledge of the local distributions of energy and stress in the supercell. The localenergy and local-stress schemes clarify these local distributions and should provide novel insights into the local structural, mechanical and chemical properties of GBs, dislocations and various defects with and without impurities.

In our technique, initially the energy density ${ }^{29}$ and stress density $^{30}$ are calculated as data on fast fourier transform mesh grids within the PAW-GGA framework ${ }^{5,7}$ via quantum materials simulator code. ${ }^{31}$ However, there exists an inherent gagedependent problem, namely the non-uniqueness for the choice of the symmetric or asymmetric forms of the kinetic terms in the energy and stress densities, originating from the presence of both forms of the kinetic operator in quantum mechanics. This problem can be settled if the energy and stress densities are integrated within some local region where the differences between the symmetric and asymmetric forms are integrated to zero. ${ }^{28}$ Such local regions that satisfy the above conditions can be determined by using the Bader partitioning of the valence charge density distribution, ${ }^{27,32}$ and the integration of the energy density (stress density) within such local regions provides local energies (local hydrostatic stresses).

Local-energy and local-stress analyses of $<110>$ symmetrical tilt grain boundaries in bcc Fe

Prior to investigating the segregation behavior of $\mathrm{Si}$, we performed systematic calculations on the structural and magnetic properties of a series of $\langle 110\rangle$ symmetrical tilt GBs (STGBs) in bcc Fe, such as $\Sigma 3$ (112), $\Sigma 3$ (111), $\Sigma 11$ (332), and $\Sigma 9$ (221), based on ab initio local-energy and local-stress analyses. Shown in Fig. 5 are a summary of the results in our previous article. ${ }^{33,} 34$ In each GB, increases in the local-energy $\Delta \varepsilon_{i}$, local tensile (positive) and compressive (negative) stresses $\sigma_{i}$, and decreases in the atomic Young's moduli $Y_{i}$ are concentrated at the interface regions, but the degree of concentration depends on each GB. Note that the atomic Young's modulus is obtained by the atomic-volume change ratio in the uniaxial stretching and compression normal to the interface for the GB supercell. ${ }^{34}$
The GB energies of these four STGBs are $0.43,1.61,1.49$, and $1.71 \mathrm{~J} / \mathrm{m}^{2}$, respectively. The $\Sigma 3(112) \mathrm{GB}$, as the most stable GB in bcc Fe, consists of stable structural units with negligible changes in the first-neighbor bond length, which is akin to stacking faults or twins, and shows very small local-energy increases and negligible atomic stresses as seen in Fig. 5(a). In contrast, the $\Sigma 9$ (221) GB shown in Fig. 5(d) is formed by the straight arrangement of 5-3 units, consisting of five-membered and three-membered rings in the $\langle 110\rangle$ projection and corresponding to edgedislocation cores. Hence, the GB structure bears a resemblance to a small-angle tilt GB formed by an edge-dislocation array. One can see an alternate distribution of compressive and tensile stresses at both edges of the 5-3 unit in a similar way to an edge dislocation. Being different from the other coincidence-site lattice (CSL) GBs ${ }^{35}$ such as $\Sigma 3$ (111) (Fig. 5(b)) and $\Sigma 11$ (332) (Fig. 5(c)), the spatial extensions of the enhanced local energies and local stresses are rather wide on both sides of the interface, resulting in a relatively high GB energy. In fact, this is remarkably different from the $\Sigma 3$ (111) and $\Sigma 11$ (332) CSL GBs for which overlapping of the cores energetically hinders the description of the GB based on an array of dislocations.

In typical CSL GBs such as $\Sigma 3$ (111) and $\Sigma 11$ (332) in Fe, we have found the general existence of two kinds of atomic sites, namely looser and tighter sites. In the $\Sigma 3$ (111) GB (Fig. 5 (b)), sites " 1 " and " 3 " are looser sites, while sites " 2 " are tighter sites. In the $\Sigma 11$ (332) GB (Fig. 5 (c)), sites " $1 \mathrm{a}$ " and " 3 " are looser sites, while sites " $1 \mathrm{~b}$ " and " 2 " are tighter sites. At a looser (tighter) site, a GB atom has a larger (smaller) atomic volume or longer (shorter) inter-atomic distances, revealing a higher (lower) local energy and a tensile (compressive) local stress. As shown in Fig. 6, it was further found that the magnetic moments at tighter sites are reduced by enhanced $d$ - $d$ covalent interactions, while those at looser sites are enhanced due to less $d$ - $d$ hybridization, leading to a larger spin polarization so as to attain a larger exchange-energy gain, as explained by the Stoner model. It should be pointed out that this shares common features with the analysis of the elastic properties discussed in the preceding section.

It should be emphasized that both looser and tighter sites are inevitably introduced at GBs when two atomic planes are encountered at a GB. This is because the atomic planes across a $\mathrm{GB}$ are by no means flat geometrically and, therefore, atomic distances are not uniform but distributed with shorter (tighter) and longer (looser) lengths than those in the bulk region. By virtue of the local-energy and local-stress schemes, the general features of the GBs in Fe stemming from the interplay among the atomic volume or interatomic distances, local energy, local stress, local elastic property, and local magnetic moment, which result in local fluctuations in the stability and elastic and magnetic properties, have been clarified for the first time. The present schemes are further extended to investigate the segregation behavior of $\mathrm{Si}$ at $\mathrm{GBs}$, which is discussed in the following section.

Local-energy and local-stress analyses of Si segregation at GBs in bcc Fe

The particular interest in the present section is the segregation behavior of Si at typical CSL STGBs such as $\Sigma 3$ (111) and $\Sigma 11$ (332) in $\mathrm{Fe}^{36}$ By applying our local-energy scheme, the segregation mechanism can be efficiently analyzed via the local-energy decomposition of the segregation energy. The segregation energy $E_{S}$ for a substitutional $\mathrm{Si}$ atom at a GB is defined as the totalenergy difference between the systems of a GB and a bulk crystal before and after the $G B$ segregation. The system before the $G B$ segregation consists of a pure $G B$ and bulk Fe crystal with one substituted $\mathrm{Si}$ atom, while the system after the GB segregation consists of a GB with a segregated $\mathrm{Si}$ atom and pure bulk $\mathrm{Fe}$ crystal. As shown in Fig. 7, the segregation energy $E_{S}$ is expressed as the sum of the following four terms via the local-energy 
(a)

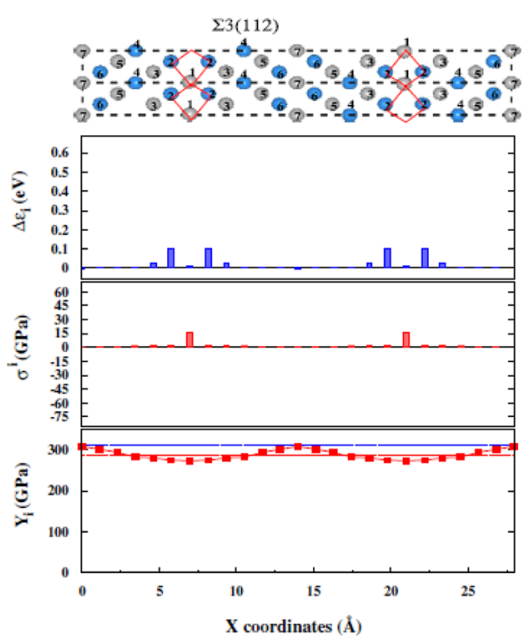

(c)
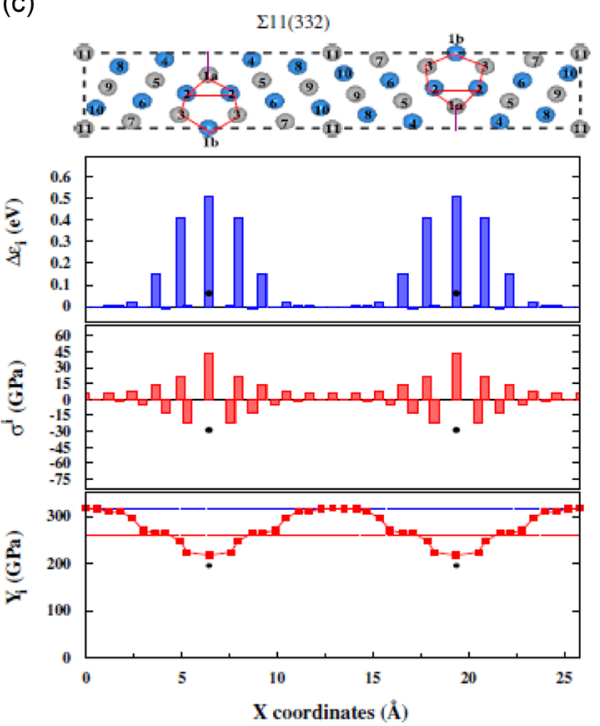

(b)
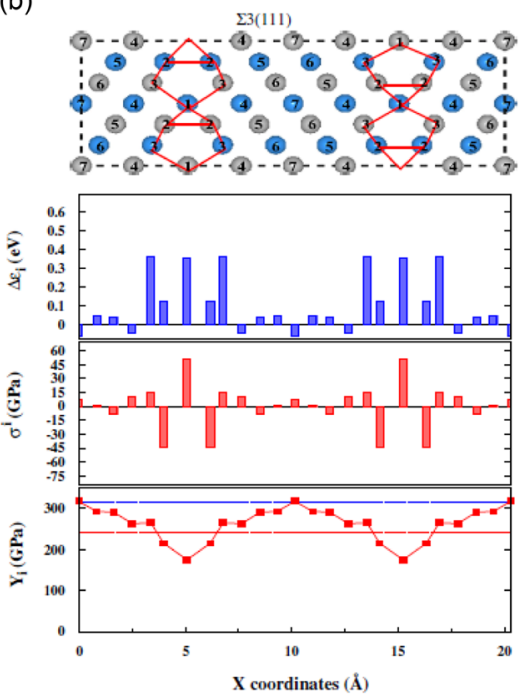

(d)

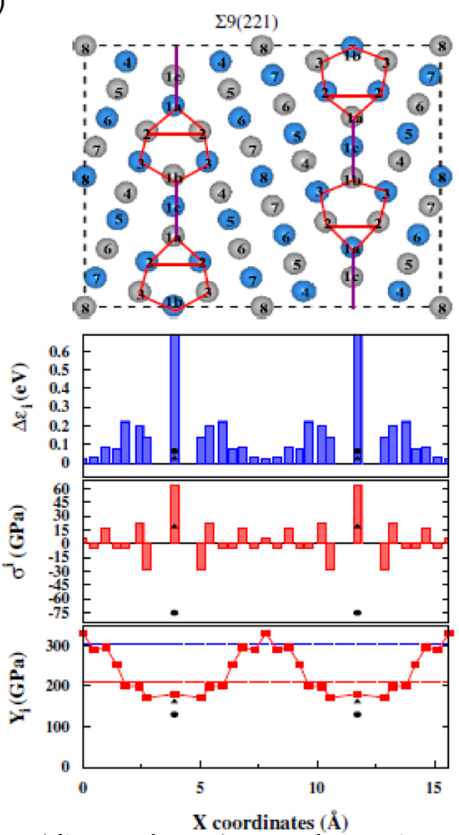

Fig. 5 Atomic energy increase $\Delta \varepsilon_{i}$ against the bulk crystal, atomic local stress (diagonal sum) $\sigma_{i}$ and atomic Young's modulus $Y_{i}$ of all the atoms in each GB supercell for four $<110>$ symmetrical tilt grain boundaries, a $\Sigma 3$ (112), b $\Sigma 3$ (111), c $\Sigma 11$ (332) and d $\Sigma 9$ (221) GBs in Fe. ${ }^{34}$ In each figure, the top panel shows the atomic configuration of the GB supercell, where two symmetric interfaces of one CSL period are contained, and structural units constituting one period are indicated by red lines. Atoms in blue and gray are those with different heights along the $<110>$ direction, and numbers at atomic sites such as " 1 " (" $1 a$ ", " $1 b$ ", or " $1 c$ "), " 2 ", " 3 ", etc. specify different and equivalent atomic sites by the symmetry. In the lower three panels (atomic energy increase $\Delta \varepsilon_{i}$, atomic local stress $\sigma_{i}$, and atomic Young's modulus $Y_{i}$ ), filled circles show the data for the atom " $1 \mathrm{~b}$ " in the $\Sigma 11$ (332) and $\Sigma 9$ (221) GBs, while filled triangles represent the data for the atom "1c" in the $\Sigma 9$ (221) GB. Red and blue horizontal broken lines in the panels of atomic Young's moduli indicate the cell-averaged Young's moduli of the GB and bulk supercells, respectively

decomposition: the stabilization of a replaced Fe atom from the GB to the bulk, $T_{1}$; the local-energy change of a Si atom from the substituted state in bulk Fe to the segregated state at a GB, $T_{2}$; the local-energy change of surrounding host Fe atoms from the pure GB to the Si-segregated GB, $T_{3}$; and the local-energy change of surrounding $\mathrm{Fe}$ atoms from the bulk Fe crystal with one substituted $\mathrm{Si}$ atom to the pure bulk crystal, $T_{4}$. The segregation mechanism can be investigated by analyzing the contribution of each term to the total value of $E_{S}$.

The $\mathrm{Si}$ segregation to looser and tighter sites with low and high densities in the $\Sigma 3$ (111) and $\Sigma 11$ (332) GBs in Fe was examined.
Sites "1" at the $\Sigma 3$ (111) GB and sites "1a" at the $\Sigma 11$ (332) GB were taken as representative looser sites, and sites " $1 \mathrm{~b}$ " at the $\Sigma 11$ (332) $\mathrm{GB}$ as tighter sites. Table 2 lists the values of $E_{S}$ and the four constituent terms for these sites, indicating a significant dependence on the segregation site and Si concentration. It is noted that looser and tighter sites show different segregation mechanisms. For the segregation at looser sites with higher local energies in the original GB configurations, the energy gain is dominated by the term $T_{1}$. In fact, for the "one-site" of the $\Sigma 3$ (111) GB and "1a-site" of the $\Sigma 11$ (332) GB in Table 2, the larger magnitudes of $T_{1}$ due to higher local energies of the sites " 1 " or " $1 a$ " in the original GBs lead 
to the segregation energy gain. It is understood that the segregation at looser sites is driven by the removal of unstable sites or bonds in the pure GBs. We may call this mechanism initialstate effects.

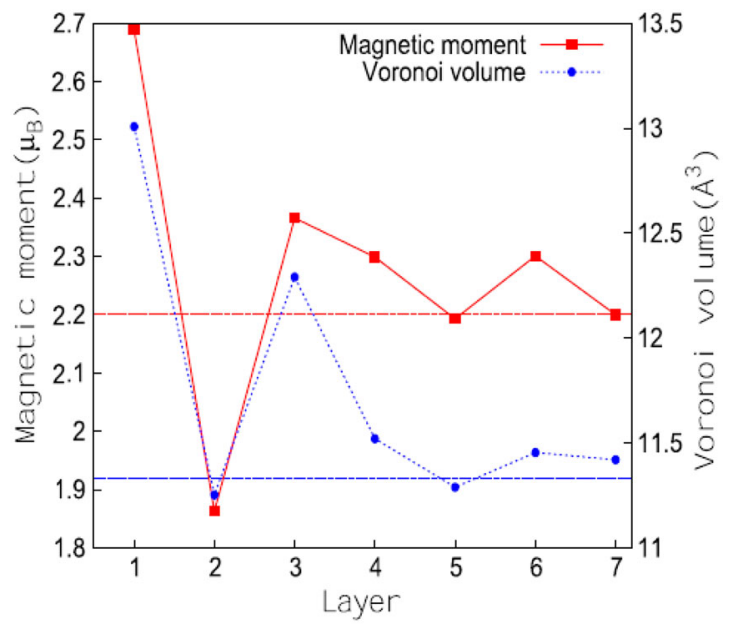

Fig. 6 Variations of local magnetic moments (in $\mu_{B}$ ) and Voronoi volumes (in ${ }^{\circ} \mathrm{A}^{3}$ ) in the atomic sites of the $\Sigma 3(111) \mathrm{GB}$ in $\mathrm{Fe}^{33}$ The horizontal axis indicates the atomic site identified in Fig. 5 (b), and each number means the atomic-layer number from the interface layer " 1 " in the configuration with mirror-plane symmetry. Red and blue horizontal broken lines show the bulk magnetic moment and the bulk Voronoi volume, respectively
The segregation at tighter sites, such as site " $1 \mathrm{~b}$ " at the $\Sigma 11$ (332) GB, can lead to much larger energy gains due to the large magnitude of $T_{2}$ or $T_{3}$, as shown in Table 2. This originates from stronger Si-Fe covalent interactions, which tend to occur locally at tighter sites in the resultant segregated configurations. This mechanism may be termed final-state effects.

When the density of segregated $\mathrm{Si}$ becomes larger, the segregation-energy gain per $\mathrm{Si}$ atom is reduced as listed in the two examples with $m=4$ in Table 2 . This is due to the interaction among neighboring $\mathrm{Si}$ atoms, which induces the local-

Table 2. Values of the segregation energy $E_{S}$, and the decomposed terms $T_{1}, T_{2}, T_{3}$ and $T_{4}$ for the Si-segregated $\Sigma 3$ (111) and $\Sigma 11$ (332) GBs in Fe in units of eV per GB supercell

\begin{tabular}{|c|c|c|c|c|c|c|c|c|}
\hline GB & $m$ & $T_{1}$ & $T_{2}$ & $T_{3}$ & $T_{4}$ & $T_{1}+T_{2}$ & $T_{3}+T_{4}$ & $E_{s}$ \\
\hline$\Sigma 3$ one-site & 2 & -0.71 & -0.19 & -2.36 & 2.70 & -0.90 & 0.34 & -0.28 \\
\hline$\Sigma 3$ two-site & 4 & -1.42 & -0.62 & -4.25 & 5.40 & -2.04 & 1.15 & -0.22 \\
\hline$\Sigma 11$ 1a-site & 2 & -1.02 & -0.04 & -1.91 & 2.70 & -1.06 & 0.79 & -0.14 \\
\hline$\Sigma 111 \mathrm{~b}$-site & 2 & -0.13 & -0.14 & -3.75 & 2.70 & -0.27 & -1.05 & -0.66 \\
\hline$\Sigma 111 a+1 b$ site & 4 & -1.15 & 0.87 & -4.45 & 5.40 & -0.28 & 0.95 & 0.17 \\
\hline
\end{tabular}

The second column shows the number of $\mathrm{Si}$ atoms $(m)$ in the GB supercell. For the $\Sigma 3$ (111) GB, "one-site" means the Si substitution for only one Fe atom at the site "1" per CSL period shown in Fig. 5 (b), while "two-site" means the Si substitution for all the sites "1" per CSL period. For the $\Sigma 11$ (332) GB, "1a-site", "1b-site" and "1a + 1b site" indicate the Si substitution at the site " $1 a$ ", the site " $1 b$ ", and both the sites per CSL period shown in Fig. 5 (c). $E_{S}$ is in units of eV/Si atom.

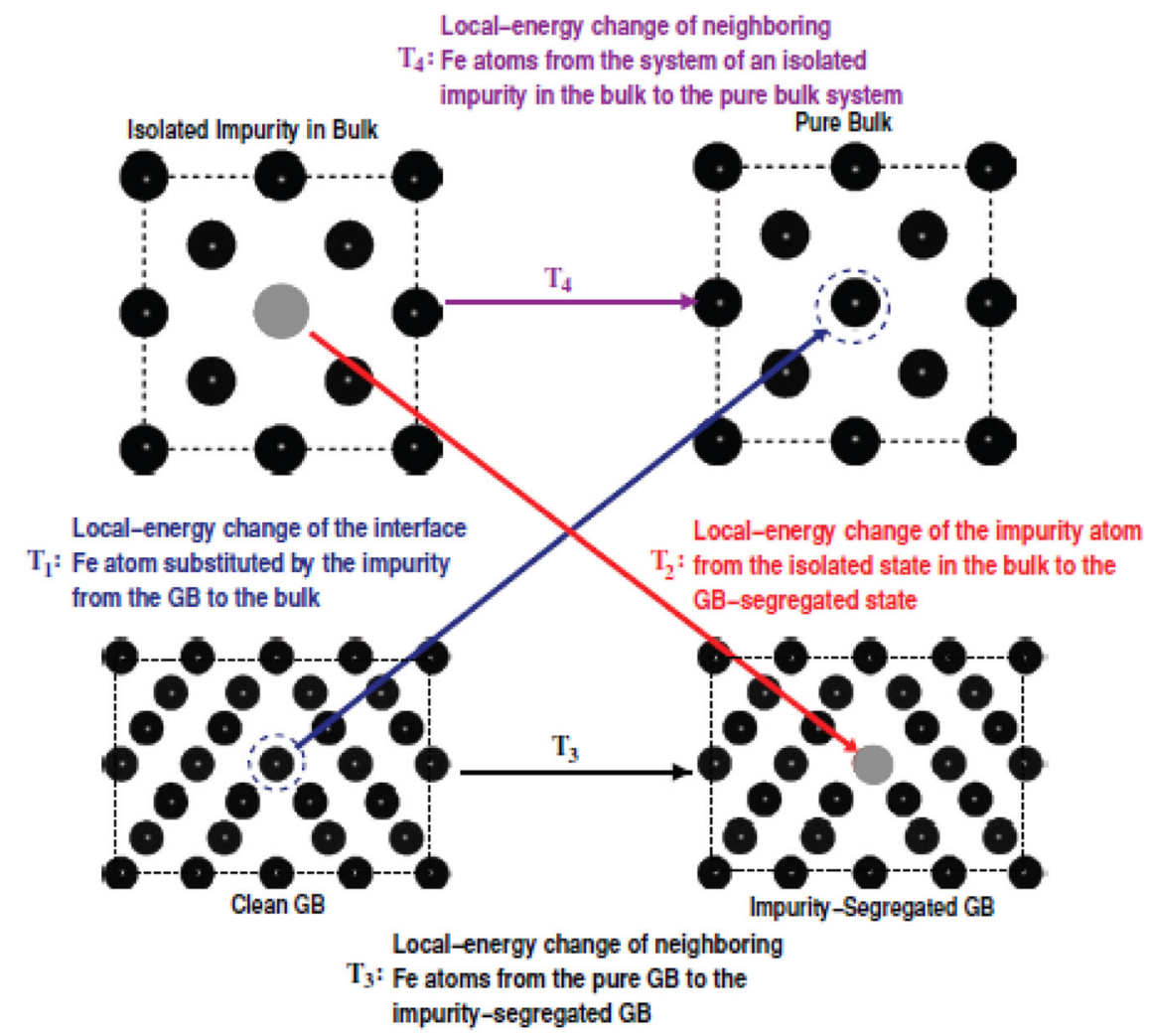

Fig. 7 Local-energy decomposition of the GB segregation energy of a substitutional impurity atom. ${ }^{36}$ The segregation energy is defined by the total-energy change from the initial system (left two structures) consisting of a pure GB and a bulk crystal containing an isolated impurity to the final system (right two configurations) consisting of an impurity-segregated GB and a pure bulk crystal. By the local-energy decomposition, the segregation energy is expressed by a sum of the four terms, $T_{1}, T_{2}, T_{3}$, and $T_{4}$ 

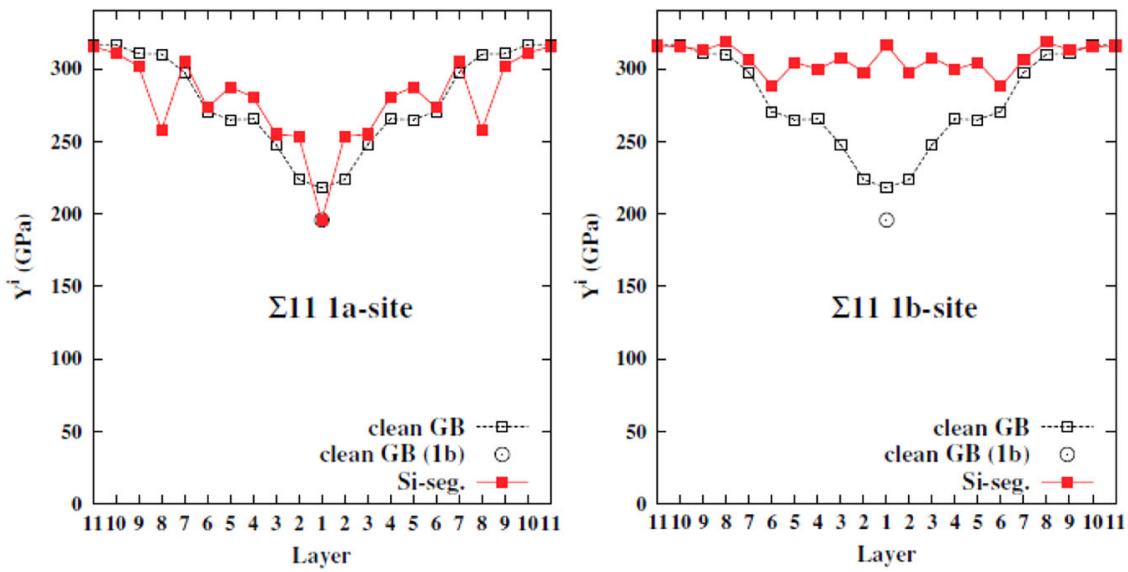

Fig. 8 Atomic Young's moduli in the pure and Si-segregated $\Sigma 11$ (332) GBs in Fe. ${ }^{36}$ Plots in red in both the figures are results of the Si substitution at " $1 a$ " and " $1 b$ ", respectively, in contrast to those of the pure GB in black. The segregation sites " $1 a$ " and " $1 b$ " are shown in Fig. 5 (c). The lowering of atomic Young's moduli at the interface is recovered by the Si substitution at the tighter site " $1 \mathrm{~b}$ " (right figure), while there is no remarkable recovery by the substitution at the looser site "1a" (left figure)

energy increases. The value of $T_{2}$ of the " $1 \mathrm{a}+1 \mathrm{~b}$ site" of the $\Sigma 11$ (332) GB is much higher than the sum of the $T_{2}$ values of the "1asite" and "1b-site". The reduction in the number of stabilized Fe atoms per $\mathrm{Si}$ atom also reduces the energy gain of $T_{3}$ as is observed in a Si dimer in bulk Fe. In fact, the magnitude of $T_{3}$ for the "two-site" of the $\Sigma 3$ (111) GB is smaller than twice that of the "one-site" of the $\Sigma 3$ (111) GB, or the magnitude of $T_{3}$ of the "1a+ $1 \mathrm{~b}$ site" of the $\Sigma 11$ (332) GB is smaller than the sum of the "1a-site" and "1b-site" of the $\Sigma 11$ (332) GB. The present calculated values of $E_{s}$ ranging from $-0.14 \mathrm{eV} / \mathrm{Si}(-13.5 \mathrm{~kJ} / \mathrm{mol})$ to $-0.66 \mathrm{eV} / \mathrm{Si}(-63.7 \mathrm{~kJ} /$ $\mathrm{mol}$ ) are consistent with the experimental values ranging from -3 $\mathrm{kJ} / \mathrm{mol}$ to $-17 \mathrm{~kJ} / \mathrm{mol}^{37}{ }^{38}$ We note that the experimental values are averaged values in GB regions obtained from various bi-crystal or polycrystalline samples of Si-doped Fe. There has been no experimental evidence of the different behavior of $\mathrm{Si}$ between looser and tighter sites. We believe that future electron microscopy observation should detect such dependences on the local environment at GBs as was shown in a recent report on oxide materials. $^{39}$

It is noted that Si segregation does not seem to induce serious weakening or embrittlement of Fe GBs, which is different from the cases of $S$ and $P$ segregation. ${ }^{40}$ The reduction in the adhesion energies of Si-segregated GBs compared to pure Fe GBs is rather small as examined in ref. 36 . It seems that Si-Fe bonds enhance local Young's moduli and suppress the interface weakening in the segregation at a tighter site as shown in Fig. 8, where the $\mathrm{Si}$ substitution at site " $1 b$ " in the $\Sigma 11$ (332) GB greatly recovers the decrease in the local Young's moduli at the interface region. The segregation at a looser site does not induce such a recovery (Fig. 8), but it also does not induce weakening or brittleness of Fe GBs. All these results should be concerned with the covalent nature of Si-Fe bonds due to strong $s p$ - $d$ hybridization, which also induces a reduction in the magnetic moments of contacting $\mathrm{Fe}$ atoms as shown clearly in ref. 36 . For GBs with much higher local Si concentrations, we do not deny the possibility of remarkable changes in the elastic and mechanical properties of GB regions observed in Fe-Si alloys, but this should be investigated in the future.

\section{DISLOCATION}

Interaction between a dislocation and a solute $\mathrm{Si}$

As described in the previous section, dislocations are typical heterogeneities in a microstructure closely related to solid- solution hardening. The main focus of the present section is single dislocation behavior. A change in the behavior of dislocations results in a change in the strength and plastic deformation properties of the whole material system, and the effect of an isolated solute atom becomes dominant when it influences the behavior of dislocations, which is realized when the area of influence of an isolated atom is comparable with the characteristic length of a dislocation.

Here, the dislocation-solute interaction and atomic details of dislocation behavior in a dilute alloy are studied using MD simulations based on interatomic potentials determined by firstprinciples electronic structure calculations. ${ }^{41,} 42$ The atomic information is then transferred to a coarse-grained kMC simulation (see supplementary information 1) to study mesoscale dislocation behavior.

There exist two types of solute atoms dissolved in the host matrix, i.e., interstitial and substitutional solute atoms. Usually interstitial solute atoms are attracted to the dislocation core and are quickly re-distributed in their vicinity because of their low energy barrier of lattice diffusion. This eventually leads to a higher solute concentration field around the dislocation. ${ }^{43}$ As a result, the effect of such a localized solute concentration field on the dislocation kinetics tends to be more effective than that of the uniform solute concentration field. Then, in the modeling of the strength and plasticity of alloys that involve such interstitial solute atoms, analyses of the dislocation motion coupled with solving the time evolution equations of the interstitial concentration around the dislocation are essential.

However, this is not the case for substitutional solute atoms like $\mathrm{Si}$ in the Fe-Si alloy system, which does not quickly diffuse like an interstitial solute atom below ambient temperature. Hence, in the modeling of such alloys, the dislocation motion in a host matrix with dispersed unmoving solute atoms is essential at usual strain rates. Here we study the strength and plasticity for the latter substitutional alloys, particularly for dilute alloys in which a random solute atom distribution is expected. We build a framework to theoretically calculate the yield stress of these alloys using atomic modeling and also kMC modeling to predict dislocation behavior in these alloys, which suggests a vein dislocation mesostructure formation instead of the formation of a cell-like structure.

It has been reported that solute Si drastically changes the mechanical properties of $\mathrm{Fe}$ as we have introduced above. The yield stress drastically increases at room temperature by the addition of a small amount of $\mathrm{Si}$, and the strength and work 
(a)

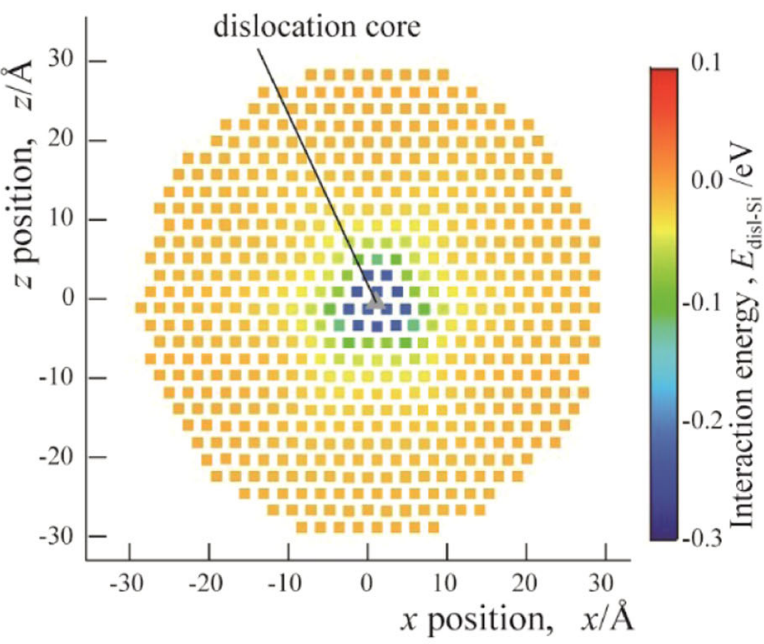

(b)

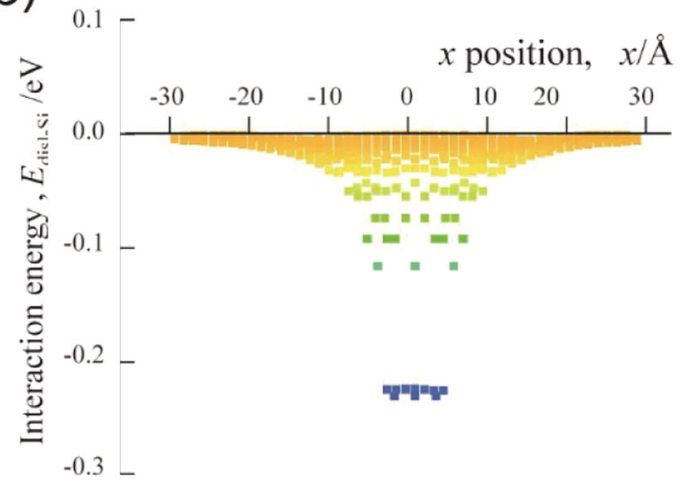

Fig. 9 Interaction energy between a straight screw dislocation and substitutional solute Si calculated based on EAM potentials; ${ }^{41}$ a distribution of the interaction energy with respect to the position of $\mathrm{Si}$ atom in the $x-z$ plane. The dislocation is located perpendicular to this plane. $\mathbf{b}$ distribution of the interaction energy with respect to the position of Si atom in $x$-direction for $z=0$, where $x:[1 \overline{12}]$ and $z:[110]$ of bcc-Fe, respectively. The dislocation line and Burgers vector $\boldsymbol{b}$ are along $y[1 \overline{1} 1]$

hardening rate also increase. The strength and plasticity of bcc-Fe are dominated by screw dislocation motion, because the energy barrier for screw dislocation motion is much higher than that of edge dislocation motion. Therefore, understanding the interaction mechanism between solute $\mathrm{Si}$ and a screw dislocation from an atomic viewpoint is the key to theoretically uncovering the underlying physics of the effects of $\mathrm{Si}$ on these mechanical properties. In the following, the kink formation process, which is the most important feature to understand various mechanical properties of Fe-Si alloy, is discussed. Then, the resolved shear stress is calculated to attempt a systematic investigation of solidsolution hardening/softening effects. Finally, kMC simulations were employed to predict potential slip systems.

\section{Kink process}

Figure 9 shows the calculated interaction energy between a straight screw dislocation and a substitutional solute $\mathrm{Si}$ atom based on the embedded atom method (EAM) interatomic potential of which the parameters were determined by a fit to the material properties of pure Fe and Fe-Si-vacancy systems obtained by first-principles calculations. ${ }^{41}$ The interaction is found to be attractive, and the interaction energy decreases when the solute Si approaches the screw dislocation core and eventually becomes constant at the dislocation core. Since our GB segregation energy analysis in the previous section suggests that the edge dislocation-Si interaction is also attractive, it can be concluded that the dislocation-Si interaction is generally attractive.

Such an intrinsic attractive interaction between the solute $\mathrm{Si}$ and the screw dislocation induces important changes in the energy barriers of kink processes, which dominate the rate of screw dislocation motion at and below ambient temperature. Figure 10 (a) and ref. 41 shows the energy profile (upper figure) and atomic configuration change of the screw dislocation (lower figure) associated with the motion on the $\{110\}$ planes along the minimum energy path (MEP) extracted by the nudged elastic band (NEB) method ${ }^{44}$ with the EAM potentials. In Fig. 10 (a, lower figure), we see that part of the screw dislocation line steps from one Peierls valley to the next with a nucleating kink pair, and the kinks move away from each other leading to a whole dislocation line shift to the next valley. The former and later processes are referred to as kink nucleation and migration, respectively. The energy barrier for kink nucleation in pure $\mathrm{Fe}$ is evaluated as 0.69 $\mathrm{eV}$ from the energy difference between states $\mathrm{A}$ and $\mathrm{C}$ in Fig. 10 (a, upper figure), while the energy barrier for kink migration is negligibly small compared with that for kink nucleation because no serration appears in the potential energy profile between states $C$ and $E$. Therefore, kink nucleation is certainly the bottleneck process of the screw dislocation motion in pure Fe. Figure 10 (c, upper figure) shows the NEB analysis of the change in energy when the screw dislocation core passes through the solute Si via the kink mechanism as shown in Fig. 10 (b, upper figure), where the solute $\mathrm{Si}$ is located in the middle nearby site 6 and the dislocation moves from site 0 to 12 . Note that the thickness of the model (dimension in the dislocation line direction) is $120 b$, where $b$ is the magnitude of the Burgers vector as shown in Fig. 10 (a, lower figure).

It is worth noting that first-principles calculations ${ }^{45}$ for pure Fe revealed that the screw dislocation has a 6-fold symmetric core structure and moves preferentially on the $\{110\}$ planes rather than the $\{112\}$ planes, and slip on $\{112\}$ planes by the combination of slips on different $\{110\}$ planes was frequently observed. Recent experimental work ${ }^{46}$ also suggested that screw dislocations move on the $\{110\}$ planes. We confirmed based on first-principles calculations that this slip plane preference is maintained even when the solute $\mathrm{Si}$ atom is located in or in the neighborhood of the dislocation core (see supplementary information 2). This $\{110\}$ slip plane preference in Fe-Si dilute alloy has also been suggested in some experimental work. ${ }^{47,48}$

Shown in Fig. 10(c) are the energy changes in the dislocation motion process for two important cases with solute $\mathrm{Si}$; case (i): kink nucleation at the nearest position to the solute $\mathrm{Si}$ in the dislocation line, and case (ii): kink nucleation at an offset position far from the solute $\mathrm{Si}$ in the dislocation line (see Fig. 10 (b, lower figure)). We also show the case without $\mathrm{Si}$ to see its effects on the energy profiles. The change in the energy barrier for kink nucleation in case (i) (lower figure in Fig. 10(c)) was $-0.15 \mathrm{eV}$ between sites 4 and 5 when the dislocation line segment approaches the solute $\mathrm{Si}$, and vice versa, while no change was found in case (ii). In fact, we examined various relative positions of the kink nucleation to the solute $\mathrm{Si}$ and found that there is almost no effect if the Si solute offset from the kinking line segment.

The solute Si may change the energy barrier for not only the kink nucleation process but also for the kink migration process. Plotted in Fig. 11(a) in red is the energy profile along the MEP for the case of a kink directly hitting the solute Si. The corresponding change in the atomic configuration is shown in Fig. 11(b). Again, the energy required for the successive kink nucleation-migration-overcoming-Si process was estimated by 
(a)
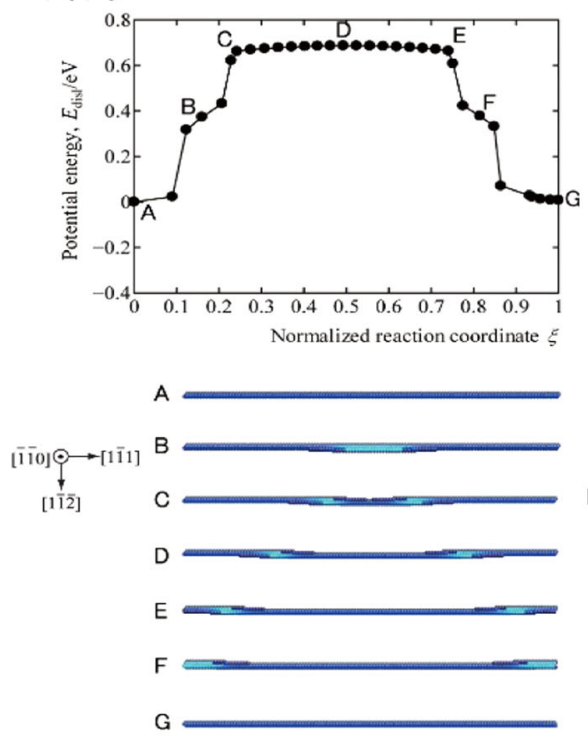

(b)
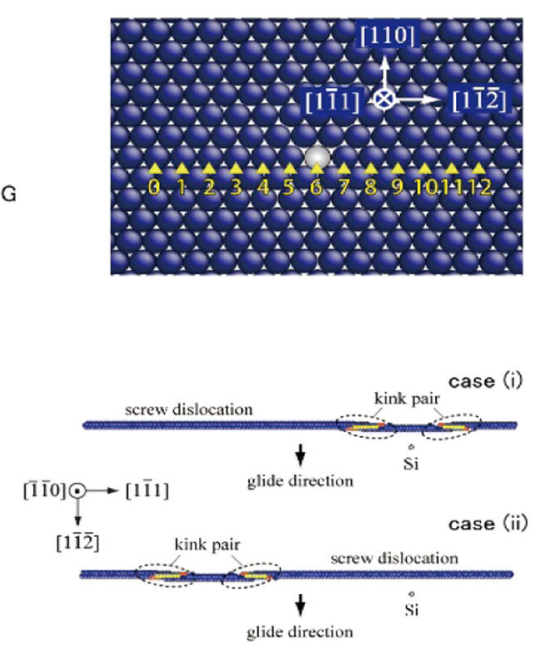

(c)
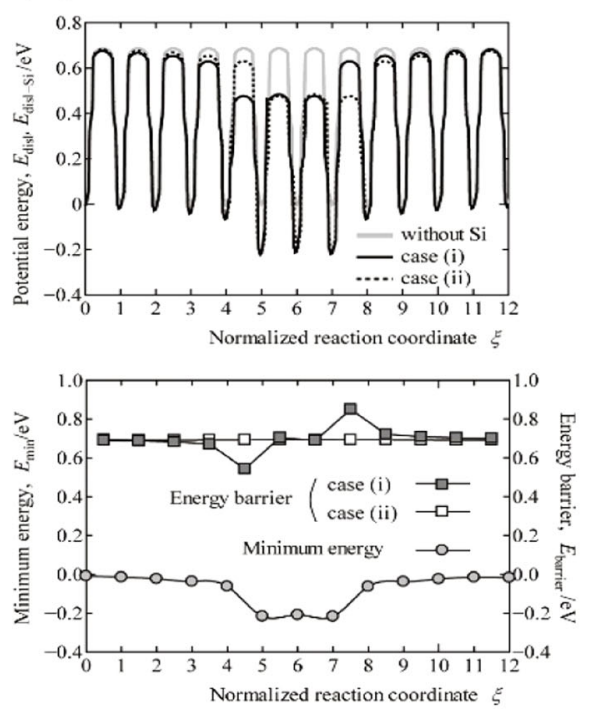

Fig. 10 a Changes in the potential energy (upper figure) and atomic configuration along MEP of screw dislocation motion (lower figure) in pure iron. b Relative configuration between screw dislocation and solute Si along the slip direction (upper figure) and the dislocation line direction (lower figure), in which Si is located near kink nucleation site (case (i)) and offset-location to kink nucleation site (case (ii)). In the lower figures, the kink pair is surrounded by dotted circle, and dislocation motion direction is represented by down arrows. c The change in the potential energy during screw dislocation motion (upper figure) and the changes in the energy barrier for kink nucleation for the cases (i) and (ii) and for the case without $\mathrm{Si}$ are summarized in the lower figure. For the case without $\mathrm{Si}$, a screw dislocation is straight and the energy corresponds to the minimum energy at each Peierls valley. ${ }^{41}$

the NEB. The black line in Fig. 11(a) is the energy profile for pure Fe reproduced from Fig. 10 (a, upper figure). Note that in order to examine only the effect of solute $\mathrm{Si}$ on the kink migration process, we initially set the position of the $\mathrm{Si}$ at the site of the dislocation core and the position of the kink nucleation in the dislocation far from the solute Si site as shown in Fig. 11(b) ${ }^{42}$ An energy barrier of $0.12 \mathrm{eV}$ appeared at $\mathrm{D}$ when the kink was overcoming the solute $\mathrm{Si}$ in the migration process (from $\mathrm{B}$ to $\mathrm{E}$ ), and this nonnegligible energy barrier for kink migration implies that the screw dislocation motion is dominated by both kink nucleation and migration in Fe-Si dilute alloys, while only by kink nucleation in pure Fe. A similar effect was found in Fe-Mn, Fe-Ni dilute alloys, while almost no effect was confirmed in $\mathrm{Fe}-\mathrm{Co}, \mathrm{Fe}-\mathrm{Cr}$ dilute alloys by first-principles calculations of the direct interaction energy between the dislocation core and solute atom (see supplementary information 3). It should be noted that when the solute Si stays even slightly away from the dislocation core, the energy profile of kink migration quickly loses the hump that appeared at $D$. It is further noted that the step height decreases with increasing distance between the solute $\mathrm{Si}$ and dislocation core. This trend can be fully described by the energy profile shown in Fig. 9 because the step height is only a function of the relative position between the dislocation line and solute Si.

\section{Calculations of CRSS}

From the calculated energy barriers for the kink nucleation and migration events, the event frequency can be evaluated based on rate theory, ${ }^{49}$ and the velocity of the screw dislocation driven by the kink nucleation and migration events at a given set of temperature, applied stress, and $\mathrm{Si}$ concentration conditions is estimated. The stress dependence of the energy barriers of these events is assumed to follow a power law, ${ }^{50}$ and the temperature dependency is ignored in this study because our target temperature is at or below ambient temperature for which a temperature effect on the energy barriers should not be significant. Using Orowan's equation, which correlates the dislocation velocity, mobile dislocation density, and Burgers vector, the CRSS was evaluated, and the temperature dependences of the calculated CRSS for pure Fe and Fe-Si are displayed in the upper figure of Fig. 12(a). At lower temperatures, the CRSS of the Fe-Si system is lower than that of pure Fe. This is because the kink nucleation frequency dominates the dislocation velocity at lower temperatures, and a decrease in the energy barrier for kink nucleation by the solute Si increases the dislocation velocity. On the other hand, at higher temperatures, the CRSS of the Fe-Si system is higher than that of pure Fe. This is due to the fact that the thermally activated kink nucleation frequency is sufficiently high, and thus kink migration governs the rate-controlling process, which drastically slows down the dislocation velocity by the solute Si. Thereby, a cross-over temperature from solid-solution softening to hardening is observed for Fe-Si alloys as is shown in the upper figure in Fig. 12(a). The cross-over temperature decreases with increasing Si concentration. This behavior of the CRSS agrees with the experimentally reported behavior ${ }^{51-53}$ presented in the lower figure of Fig. 12(a). We further made a direct comparison of the calculated CRSS with the experimental values for pure Fe and Fe-2\%Si alloys in Fig. 12(b). Figure 12(c) shows the CRSSs with respect to the Si concentration for both calculated (upper figure) and experimentally reported (lower figure) values at various temperatures. At lower temperatures, both the calculated and experimentally reported CRSSs initially decrease with increasing $\mathrm{Si}$ concentration then increase, although the CRSSs monotonically increase at higher temperatures. Figure 12(d) provides a direct comparison of the calculated and experimental CRSSs for two temperatures, 123 and $373 \mathrm{~K}$. These confirm the solid-solution softening with lower Si concentrations and solid-solution hardening with higher $\mathrm{Si}$ concentrations at lower temperatures. Note that the above analysis ignores the non-Schmid effect on bcc screw dislocations because it should be very small in the case of Fe. ${ }^{54}$ 
(a)

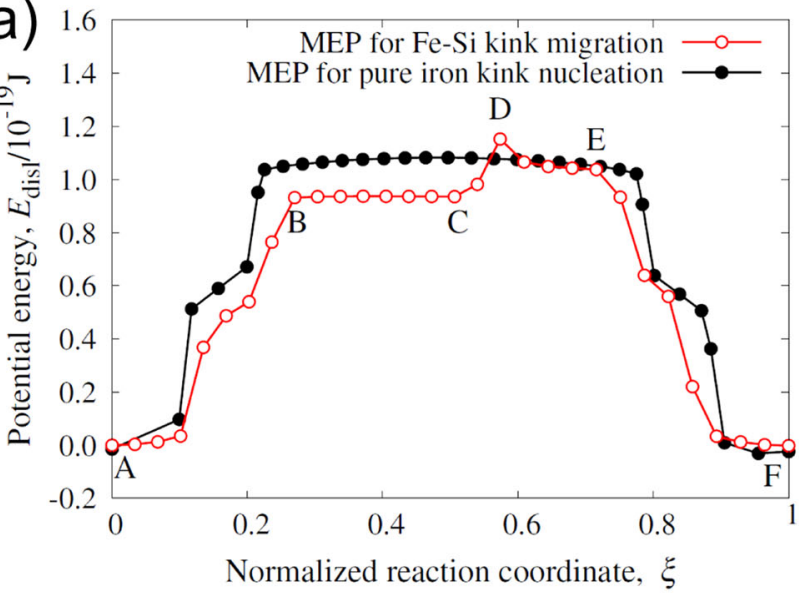

(b)
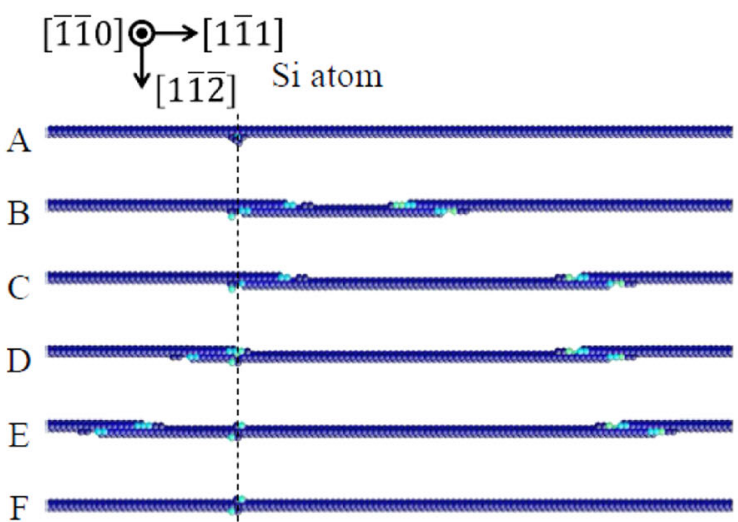

Fig. 11 a The change in the potential energy profile during screw dislocation motion along MEP in pure $\mathrm{Fe}$ and $\mathrm{Fe}-\mathrm{Si}$, in which $\mathrm{Si}$ is located so as to interact with screw dislocation during kink migration process as shown in the figure (b). b The screw dislocation motion along MEP via kink mechanism under the effect of solute $\mathrm{Si}$, of which position is represented by a dashed vertical line. ${ }^{42}$ The atoms are colored by the central symmetry

\section{Preferential slip systems studied by kMC simulations}

The present theoretical framework predicting the dislocation velocity successfully describes the underlying physics of the yield strength in dilute bcc alloys. However, the framework does not answer the question why the most preferable slip system often changes by the addition of a solute atom. ${ }^{48,55}$ In fact, in the Fe-Si dilute alloys, the $\{110\}<111>$ slip system becomes the most preferable, ${ }^{48,55}$ while the $\{110\}<111>$ and $\{112\}<111>$ slip systems are equally preferable in pure Fe. To answer this question, we constructed a spatiotemporal coarse-grained $\mathrm{kMC}^{56}$ based on the energy barriers of kink nucleation and migration with and without solute $\mathrm{Si}$. In this $\mathrm{kMC}$, the screw dislocation is modeled by the collection of many line segments. Each line segment is a screw dislocation line, and two neighboring segments make a kink if their position is different in the slip direction. The size of each segment is chosen to be $10 b$, which is smaller than the typical length of a nucleated kink, $40 b .^{45}$ The line segment can move on any one of $\{110\}$ planes, making a new kink pair (two kink segments) or a kink segment. The nucleated kink may migrate along the dislocation line and make a jog if the kink on a slip plane hits the other kink with the opposite edge component in the other slip plane. A stochastic process is employed in the calculations of the interaction between the Si solutes and the screw dislocation. Note again that the existence of a $\mathrm{Si}$ solute atom does not influence the fundamental slip plane, which is always $\{110\}$ as mentioned above, but it does changes the kink nucleation frequency and average kink migration velocity along the dislocation line. The kMC can describe the time evolution of the shape of the screw dislocation line including the jog formation and cross-slip events.

Figure 13(a) shows snapshots of a screw dislocation with and without Si that is subject to resolved shear stresses at $T=100 \mathrm{~K}$ acting in the $<111>$ direction on the $\{110\}$ planes (case (i)) and in the $<111>$ direction on the $\{112\}$ planes (case (ii)). In the case of pure Fe (left figures in Fig. 13(a) (i) and (ii)), the kink migration rate is much higher than the kink nucleation rate as seen in Fig. 11(a), and thus a screw dislocation with a typical length of $\sim 10^{3} \mathrm{~nm}$ may usually have only one kink pair at the same time. Hence, as soon as kink nucleation occurs, the whole dislocation line moves from one Peierls valley to the next accompanying the fast kink migration along the dislocation line. Therefore, cross slip from one $\{110\}$ plane to other $\{110\}$ planes may frequently occur. The cross slip of screw dislocations was actually observed in the kMC simulation, particularly in case (ii) with shear stress acting on the $\{112\}$ planes. On the other hand, in the case of Fe-Si alloys, as was discussed in the yield stress analysis above, the kink nucleation rate is enhanced to the same order of the kink migration rate, which leads to multiple kink nucleation on different $\{110\}$ planes at the same time, particularly in case (ii), and this results in frequent kink collisions by their lateral motion along the screw dislocation line. The collisions of kinks on different $\{110\}$ planes form many jogs in the screw dislocation as shown in Fig. 13(a). Our kMC simulation clearly suggests that solute Si drastically promotes the jog formation in case (ii) (see Fig. 13(b)).

It was also found that the jaggy shape of a screw dislocation involving a high density of jogs effectively suppresses the cross slip of the whole dislocation line, implying that solute Si has a significant effect on the mobility of screw dislocations. It has been reported ${ }^{55}$ that solute Si changes the work hardening rate and fatigue properties by changing the dislocation substructure. In fact, dislocations distribute in a more planar manner in the $\{110\}$ planes, and the dislocation substructure becomes a vein structure, which is composed of alternate high and low dislocation density regions. The kMC simulation offers one of the possible mechanisms of these experimental observations. In the case of pure Fe, the screw dislocation can easily be crossslip triggered by the local stress like the back stress of immobile dislocations or other obstacles ahead of it. Thus, the dislocation may sidestep these immobile dislocations if there is free space beside them. A large-scale 3D dislocation dynamics simulation $^{57}$ suggests a cell structure formation due to the easy cross slip, which is also observed as a cell-like pattern in the plane perpendicular to the Burgers vector. ${ }^{55}$ On the other hand, in the case of Fe-Si alloy, since the cross slip becomes difficult, a dislocation stuck to other immobile dislocations leads to work hardening. Eventually, dislocations may form the vein structure, which is usually observed in dilute $\mathrm{Fe}-\mathrm{Si}$ alloys as mentioned above. We believe further large-scale 3D dislocation dynamics simulations based on our kMC results may directly demonstrate the vein structure formation.

\section{SUMMARY}

Recent developments in computational materials science enable us to investigate the electronic origins of the mechanical properties of a given alloy system. By taking Fe-rich Si alloy as an example, we employed computational tools to demonstrate how the origins of the peculiar mechanical properties of this system can be clarified. These included the (i) combined magneto- 
(a)
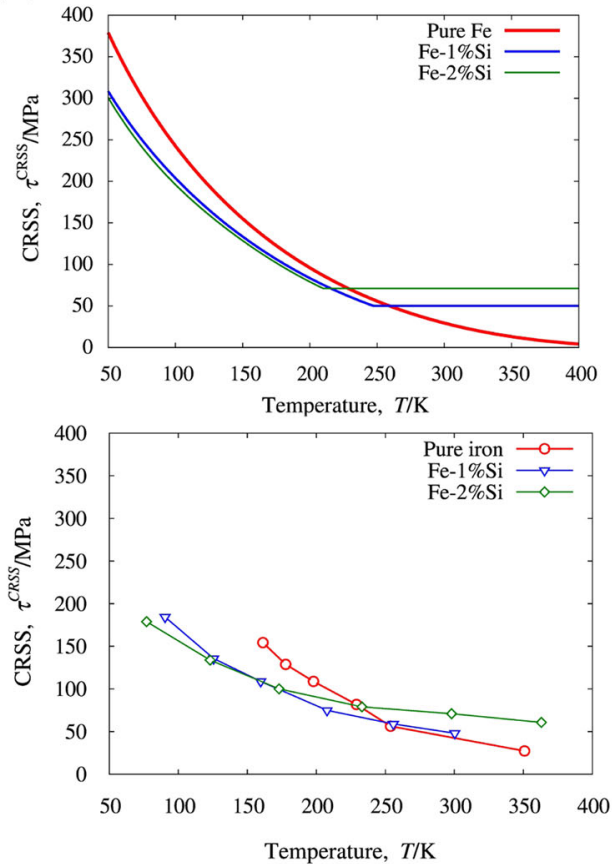

(c)
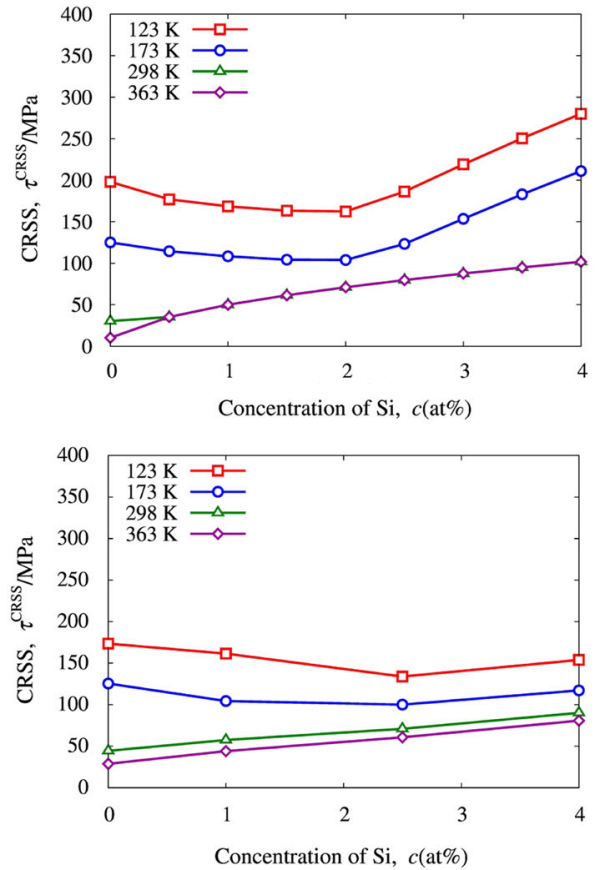

(b)
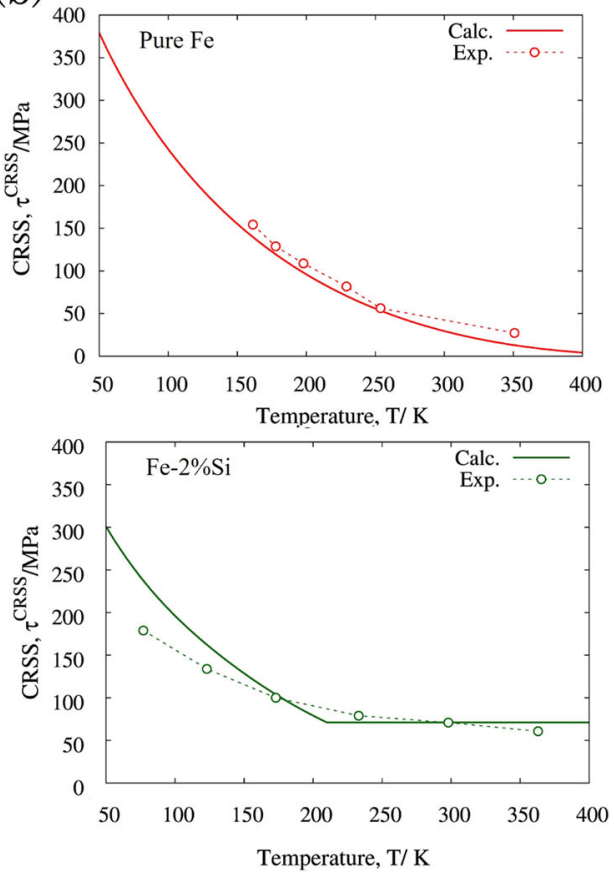

(d)
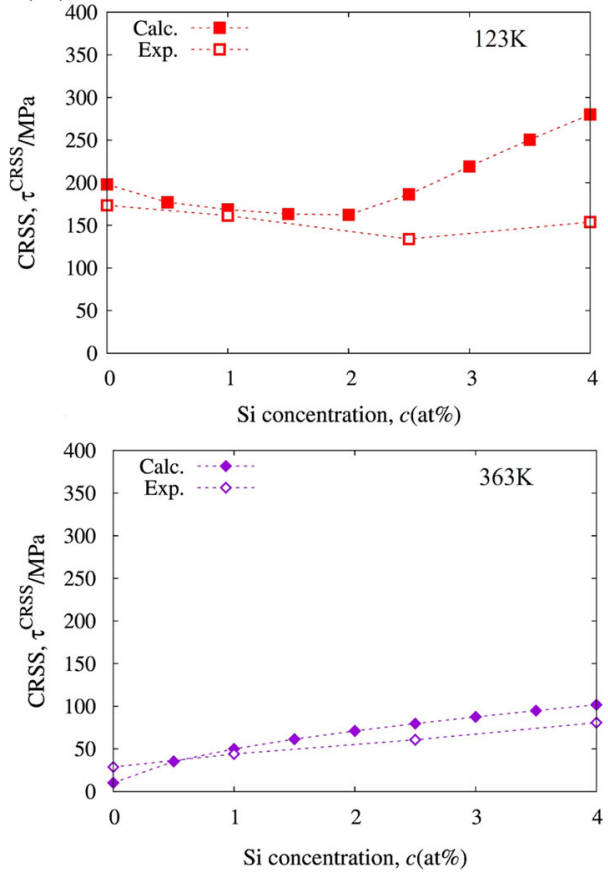

Fig. 12 a Theoretically estimated (upper figure) and experimentally reported (lower figure) CRSSs of pure Fe and Fe-Si as a function of temperature. In the upper figure, CRSSs of Fe-Si system are constant at high temperature due to athermal component of CRSS. In the lower figure, experimental values of pure $\mathrm{Fe}, \mathrm{Fe}-1 \mathrm{at} \% \mathrm{Si}$ and Fe-2at\%Si are referenced from ref. $51-53$, respectively. $\mathbf{b}$ Direct comparisons of the calculated CRSS with experimental ones for pure Fe (upper) and Fe-2\%Si (lower) alloys. c CRSSs with respect to the Si concentration for calculated (upper figure) and experimentally reported (lower figure) results at various temperatures. $\mathbf{d}$ Direct comparisons between calculated and experimental CRSSs at two temperatures, $123 \mathrm{~K}$ and $373 \mathrm{~K}$

volume effect and ordering tendency, leading to ductile-brittle behavior with a change in the concentration, (ii) local stress and energy distributions around grain boundaries, enhancing/suppressing segregation of $\mathrm{Si}$, and (iii) kink nucleation and propagation on a screw dislocation, dominating solid-solution hardening and softening and preferential selection of slip systems. The present study is regarded as a precursor to more sophisticated multiscale calculations to predict stress-strain behavior. Such a challenge has been already attempted by the authors' groups and the results will be reported elsewhere. 
(a)

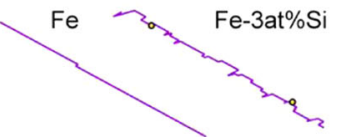

(i)

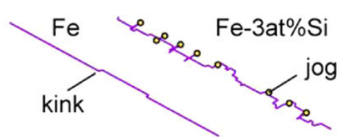

(ii) (b)

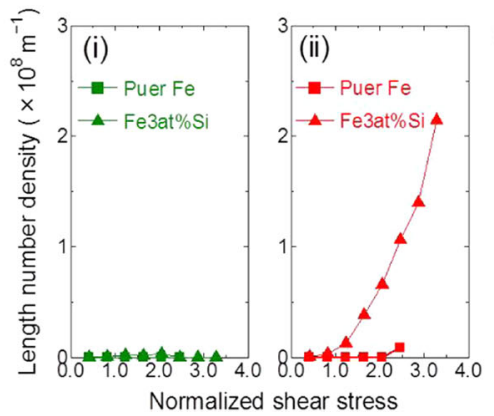

(c)

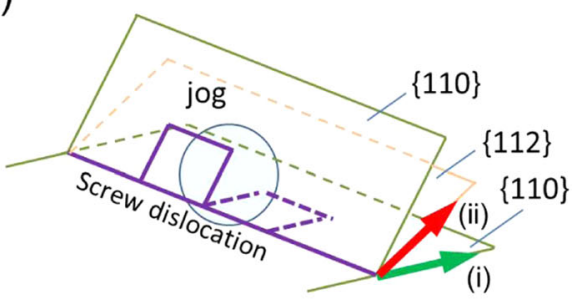

Fig. 13 a Shape of a screw dislocation line for pure iron and Fe-3at\%Si alloy obtained from kMC simulations at $100 \mathrm{~K}$ under two different stress conditions; (i) shear stress acting on $\{110\}$ plane to $\langle 111\rangle$ direction, and (ii) on $\{112\}$ plane to $\langle 111\rangle$ direction. Closed circles represent jogs formed by collision of two kinks. b Length number density of jogs formed in the KMC simulation during 100,000 kMC steps at $T=100 \mathrm{~K}$ for pure iron and Fe-3at\%Si alloy under different stress levels of the cases (i) and (ii). The shear stress is normalized by the shear modulus $\mathrm{C}_{44}=122 \mathrm{GPa}$ of pure iron. c Schematic illustration of a jog formation on bcc screw dislocation caused by collision of two kinks on different $\{110\}$ planes

\section{ACKNOWLEDGEMENT}

The present study was supported by the JST Industry-Academia Collaborative Programs, "Materials Strength from Hamiltonian", and by the Elements Strategy Initiative for Structural Materials (ESISM) through MEXT, Japan. This study was partly supported by a Grant-in-Aid for Scientific Research on Innovative Area "Bulk Nanostructured Metals" and by the Computational Materials Science Initiative (CMSI), MEXT, Japan. We also acknowledge the Supercomputer Center at IMR, Tohoku University. A part of this research used computational resources of the $\mathrm{K}$ computer provided by the RIKEN Advanced Institute for Computational Science through the HPCI System Research project (Project ID:hp130016, hp140233, hp150235). Finally, the authors expresses thanks to Ms. N. Kadowaki and Mr. R. Yamada for careful assistance in preparing the manuscript.

\section{COMPETING INTERESTS}

The authors declare no competing interest.

\section{REFERENCES}

1. Saengdeejing, A., Chen, Y., Suzuki, K., Miura, H. \& Mohri, T. First-principles study on the dilute $\mathrm{Si}$ in bcc Fe: electronic and elastic properties up to 12.5 at.\%Si. Comput. Mat. Sci. 70, 100-106 (2013).

2. Mohri, T. Multiscale approach to materials designing "from Hamiltonian to strong alloys". Bull. Iron and Steel Ins. Jpn. 17, 842-847 (2012).

3. Ushioda, K., Takahashi, J., Takebayashi, S., Maeda, D., Hayashi, K. \& Abe, Y. R. Challenges toward the further strengthening of sheet steel. in Avanced Steel (ed. Weng, Y. ) 229-240 (Springer-Verlag, 2011).

4. Kawasaki, Y., Nakagaito, T., Kaneko, S., Takagi, S. \& Matsuoka, S. Effect of Si on work hardening behavior in IF steels. in Proc. 2nd Inte. Symp. Steel Sci. 241-244 (ISSS 2009).

5. Kresse, G. \& Joubert, D. From ultrasoft pseudopotentials to the projector augmented-wave method. Phys. Rev. B. 59, 1758-1775 (1999).

6. Kresse, G. \& Furthmüller, J. Efficiency of ab-initio total energy calculations for metals and semiconductors using a plane-wave basis set. Comput. Mat. Sci. 6, 15-50 (1996).

7. Blochl, P. E. Projector augmented-wave method. Phys. Rev. B. 50, 17953-17979 (1994).

8. Perdew, J. P., Burke, K. \& Ernzerhof, M. Generalized gradient approximation made simple. Phys. Rev. Lett. 77, 3865-3868 (1996).

9. Connolly, J. W. D. \& Williams, A. R. Density-functional theory applied to phase transformations in transition-metal alloys. Phys. Rev. B. 27, 5169-5172 (1983).

10. van de Walle, A., Asta, M. \& Ceder, G. The alloy theoretic automated toolkit: a user guide. CALPHAD. 26, 539-553 (2002).

11. Kikuchi, R. A theory of cooperative phenomena. Phys. Rev. 81, 988-1003 (1951).

12. Kikuchi, R. Superposition approximation and natural iteration calculation in cluster variation method. J. Chem. Phys. 60, 1071-1080 (1974).

13. Le Page, Y. \& Saxe, P. Symmetry-general least-squares extraction of elastic data for strained materials from ab initio calculations of stress. Phys. Rev. B. 65, 104104 1-104104-14 (2002).

14. Simmons, G. \& Wang, H. Single Crystal Elastic Constants and Calculated Aggregate Properties A Handbook. (The MIT Press, 1971).

15. Bozorth, R. M. Ferromagnetism (Van Nostrand, 1951).

16. Machová, A. \& Kadec ková, S. Elastic constants of iron-silicon alloy single crystals. Czech. J. Phys. 27, 555-563 (1977).
17. Ota, K. Development of Siteel (high-strength Fe-Si alloy containing 3.5-7\%Si). Iron and Steel. 56, 614-621 (1970).

18. Chen, Y., Saengdeejing, A. \& Mohri, T. First-principles study of Ni doping effect on mechanical properties of dilute Fe-Si alloy. in Proc. PTM 2015 911-912 (TMS, 2015).

19. Chen, Y., Saengdeejing, A. \& Mohri, T. Mechanical properties of dilute Si in Fe-Si alloy and Ni-doping effect: an integrated study based on first-principles. in Proc. 2015 Inte. Sym. Multi-scale Mode. Sim. Mat. 24 (Chinese Materials Research Society (C-MRS), 2015).

20. Pugh, S. F. Relations between the elastic moduli and the plastic properties of polycrystalline pure metals. Phil. Mag. 45, 823-843 (1954).

21. Schlosser, W. F. The magnetovolume effect in Fe. Phys. stat. sol. (a) 22, K219-K222 (1974).

22. Khmelevska, T., Khmelevskyi, S., Ruban, A. V. \& Mohn, P. Magnetism and origin of non-monotonous concentration dependence of the bulk modulus in Fe-rich alloys with Si, Ge and Sn: a first-principles study. J. Phys. Condens. Mat. 18, 6677-6689 (2006).

23. Ohnuma, I., Abe, S., Shimenouchi, S., Omori, T., Kainuma, R. \& Ishida, K. Experimental and Thermodynamic Studies of the Fe-Si Binary System. ISIJ Int. 52, 540-548 (2012).

24. Liang, Y. F., Shang, S. L., Wang, J., Wang, Y., Ye, F., Lin, J. O., Chen, G. L. \& Liu, Z. K. First-principles calculations of phonon and thermodynamic properties of $\mathrm{Fe}-\mathrm{Si}$ compounds. Intermetallics. 19, 1374-138 (2011).

25. Olsson, P., Klaver, T. P. C. \& Domain, C. Ab initio study of solute transition-metal interactions with point defects in bcc Fe. Phys. Rev. B. 81, 054102-1-054102-12 (2010).

26. Liu, C., Asato, M., Fujima, N. \& Hoshino, T. Full-potential KKR calculations for point defect energies in Fe-based dilute alloys, based on the generalized-gradient approximation. Mater. Trans. 54, 1667-1672 (2013).

27. Wang, H., Kohyama, M., Tanaka, S. \& Shiihara, Y. Ab initio local energy and local stress: application to tilt and twist grain boundaries in $\mathrm{Cu}$ and Al. J. Phys.: Condens. Matter. 25, 305006 (2013). (13 pages).

28. Shiihara, Y., Kohyama, M. \& Ishibashi, S. Ab initio local stress and its application to Al (111) surfaces. Phys. Rev. B. 81, 075441-1-075441-11 (2010).

29. Chetty, N. \& Martin, R. M. First-principles energy density and its applications to selected polar surfaces. Phys. Rev. B. 45, 6074-6088 (1992).

30. Filippetti, A. \& Fiorentini, V. Theory and applications of the stress density. Phys. Rev. B. 61, 8433-8442 (2000).

31. Ishibashi, S., Tamura, T., Tanaka, S., Kohyama, M. \& Terakura, K. Ab initio calculations of electric-field-induced stress profiles for diamond/c-BN (110) superlattices. Phys. Rev. B. 76, 153310-1-153310-4 (2007).

32. Yu, M., Trinkle, D. R. \& Martin, R. M. Energy density in density functional theory: application to crystalline defects and surfaces. Phys. Rev. B. 83, 115113-1-11511310 (2011).

33. Kr Bhattacharya, S., Tanaka, S., Shiihara, Y. \& Kohyama, M. Ab initio study of symmetrical tilt grain boundaries in bcc Fe: structural units, magnetic moments, interfacial bonding, local energy and local stress. J. Phys. Condens. Matter. 25, 135004 (2013). (14 pages).

34. Kr Bhattacharya, S., Tanaka, S., Shiihara, Y. \& Kohyama, M. Ab initio perspective of the $<110>$ symmetrical tilt grain boundaries in bcc Fe: application of local energy and local stress. J. Mater. Sci. 49, 3980-3995 (2014).

35. Sutton, A.P. \& Balluffi, R.W. in Interfaces in Crystalline Materials (Oxford, 1995).

36. Kr Bhattacharya, S., Kohyama, M., Tanaka, S. \& Shiihara, Y. Si segregation at Fe grain boundaries analyzed by ab initio local energy and local stress. J. Phys.: Condens. Matter. 26, 355005 (2014). (18 pages). 
37. Lejcek, P. et al. (eds) Grain Boundary Segregation in Metals (Springer, 2010).

38. Liu, C.M., Abiko, K. \& Kimura, H. Effect of silicon on the grain boundary segregation of phosphorus and the phosphorus induced intergranular fracture in high purity Fe-Si-P alloys. in Proc. 8th Int. Conf. Str. Met. Alloys (Tampere) 1101-1106 (Pergamon, 1988).

39. Sato, Y., Roh, J.-Y. \& Ikuhara, Y. Grain-boundary structural transformation induced by geometry and chemistry. Phys. Rev. B. 87, 140101-1-140101-4 (2013).

40. Yamaguchi, M., Nishiyama, Y. \& Kaburaki, H. Decohesion of iron grain boundaries by sulfur or phosphorous segregation: first-principles calculations. Phys. Rev. B. 76, 035418-1-035418-5 (2007).

41. Wakeda, M., Kimizuka, H. \& Ogata, S. Atomistic study of interaction between screw dislocation and Si atom in Fe-Si alloy. J. Japan Inst. Met. Mater 77, 409-414 (2013).

42. Shinzato, S., Wakeda, M. \& Ogata, S. Theoretical prediction of macroscopic yield strength for Fe alloy based on atomistic study. J. Japan Inst. Met. Mater. 80, 197-205 (2016).

43. Ventelon, L., Lüthi, B., Clouet, E., Proville, L., Legrand, B., Rodney, D. \& Willaime, F. Dislocation core reconstruction induced by carbon segregation in bcc iron. Phys. Rev. B. 91, 220102(R)-1-220102(R)-5 (2015).

44. Henkelman., G. \& Jónsson, H. Improved tangent estimate in the nudged elastic band method for finding minimum energy paths and saddle points. J. Chem. Phys. 113, 9978-9985 (2000).

45. Itakura, M., Kaburaki, H. \& Yamaguchi, M. First-principles study on the mobility of screw dislocations in bcc iron. Acta. Mater. 60, 3698-3710 (2012).

46. Caillard, D. Kinetics of dislocations in pure Fe. Part I. In situ straining experiments at room temperature. Acta. Mater. 58, 3493-3503 (2010).

47. Weinberger, C. R., Boyce, B. L. \& Battaile, C. C. Slip planes in bcc transition metals. Int. Mater. Rev. 58, 296-314 (2013).

48. Barrett, C. S., Ansel, G. \& Mehl, R. F. Slip, twinning and cleavage in iron and silicon ferrite. Trans. ASM. 25, 702-736 (1937).

49. Argon, A. S. Strengthening mechanisms in crystal plasticity. (Oxford University Press: New York, 2008).

50. Kocks, U. F., Argon, A. S. \& Ashby, M. F. Thermodynamics and kinetics of slip. Prog. Mat. Sci 19, 1-291 (1975).
51. Okazaki, K. J. Solid-solution hardening and softening in binary iron alloys. Mater. Sci. 31, 1087-1099 (1996).

52. Kitajima, K., Aono, Y., Abe, H., \& Kuramoto, E. Solid solution hardening and softening in iron alloy single crystals between $4.2 \mathrm{~K}$ and $300 \mathrm{~K}$. In Proc. 5th Int. Conf. Stre. Meta. Alloys (ed. Haasen, P., Gerold, V., Kostorz, G.) 2, 965-970 (Pergamon Press, 1979).

53. Chen, Y. T., Atteridge, D. G. \& Gerberich, W. W. Plastic flow of Fe-binary alloys-I. A description at low temperatures. Acta Metall. 29, 1171-1185 (1981).

54. Dezerald, L., Rodney, D., Clouet, E., Ventelon, L. \& Willaime, F. Plastic anisotropy and dislocation trajectory in BCC metals. Nat. Commun. 7, 11695-1-11695-7 (2016).

55. Ushioda, K., Goto, S., Komatsu, Y., Hoshino, A. \& Takebayashi, S. Evolution of dislocation structure and fatigue crack behavior in Fe-Si alloys during cyclic bending test. Tetsu-to-Hagané. 94, 321-330 (2008).

56. Deo, C. S., Srolovitz, D. J., Cai, W. \& Bulatov, V. V. Kinetic monte carlo method for dislocation migration in the presence of solute. Phys. Rev. B. 71, 0141061-014106-12 (2005).

57. Déprésab, C., Robertson, C. F. \& Fivel, M. C. Low-strain fatigue in AISI 316L steel surface grains: a three-dimensional discrete dislocation dynamics modelling of the early cycles I. dislocation microstructures and mechanical behavior. Phil. Mag. 84, 2257-2275 (2004).

\section{cc) (i)}

This work is licensed under a Creative Commons Attribution 4.0 International License. The images or other third party material in this article are included in the article's Creative Commons license, unless indicated otherwise in the credit line; if the material is not included under the Creative Commons license, users will need to obtain permission from the license holder to reproduce the material. To view a copy of this license, visit http://creativecommons.org/licenses/by/ $4.0 /$

(c) The Author(s) 2017

Supplementary Information accompanies the paper on the npj Computational Materials website (doi:10.1038/s41524-017-0012-4). 